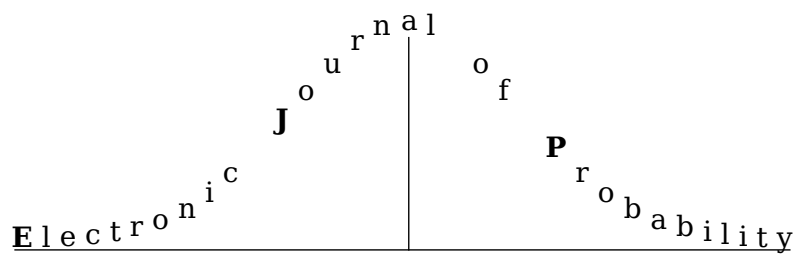

Electron. J. Probab. 26 (2021), article no. 27, 1-35.

ISSN: 1083-6489 https://doi.org/10.1214/21-EJP586

\title{
Higher order fluctuation fields and orthogonal duality polynomials*
}

\author{
Mario Ayala $^{\dagger} \quad$ Gioia Carinci ${ }^{\ddagger} \quad$ Frank Redig ${ }^{\S}$
}

\begin{abstract}
Inspired by the works in [2] and [11] we introduce what we call $k$-th-order fluctuation fields and study their scaling limits. This construction is done in the context of particle systems with the property of orthogonal self-duality. This type of duality provides us with a setting in which we are able to interpret these fields as some type of discrete analogue of powers of the well-known density fluctuation field. We show that the weak limit of the $k$-th order field satisfies a recursive martingale problem that corresponds to the SPDE associated with the $k$ th-power of a generalized Ornstein-Uhlenbeck process.
\end{abstract}

Keywords: fluctuation fields; higher-order fields; orthogonal polynomials; self-duality. MSC2020 subject classifications: $60 \mathrm{~K} 35$; $35 \mathrm{~K} 55$.

Submitted to EJP on May 10, 2020, final version accepted on January 30, 2021.

\section{Introduction}

In the context of interacting particle systems with a conserved quantity (such as the number of particles) in $[6,12]$ one studies the time-dependent density fluctuation field

$$
\mathscr{X}^{(n)}\left(\varphi, \eta\left(n^{2} t\right)\right)=\frac{1}{n^{d / 2}} \sum_{x \in \mathbb{Z}^{d}} \varphi(x / n)\left(\eta_{x}\left(n^{2} t\right)-\rho\right) .
$$

Here $\varphi$ denotes a test-function, and $\eta_{x}$ the number of particles at site $x \in \mathbb{Z}^{d}$. The quantity $\mathscr{X}^{(n)}\left(\varphi, \eta\left(n^{2} t\right)\right)$ is then considered as a random time-dependent (Schwartz) distribution. In a variety of models with particle number conservation (such as zerorange processes, simple exclusion processes, etc.), this time-dependent field is proved

\footnotetext{
${ }^{*}$ M. Ayala acknowledges financial support from the Mexican Council on Science and Technology (CONACYT) via the scholarship 457347.

${ }^{\dagger}$ Delft University of Technology, The Netherlands.

E-mail: m.a.ayalavalenzuela@tudelft.nl

${ }^{\ddagger}$ University of Modena and Reggio Emilia, Italy.

E-mail: gioia.carinci@unimore.it

${ }^{\S}$ Delft University of Technology, The Netherlands.

E-mail: f.h.j.redig@tudelft.nl
} 
to converge, at equilibrium, to a stationary infinite-dimensional Ornstein-Uhlenbeck process. This scaling limit behavior of the density fluctuation field can be thought of as a generalized space-time central limit theorem.

The usual strategy of proof (see e.g. Chapter 11 of [12]) is to start from the Dynkin martingale associated to the density field and prove convergence of the drift term via the Boltzmann-Gibbs principle (the drift term becomes in the scaling limit a function of the density field), and convergence of the noise term via characterization of its quadratic variation (which becomes deterministic in the scaling limit). This then eventually leads to the informally written SPDE

$$
d \mathscr{X}_{t}=D \Delta \mathscr{X}_{t}+\sigma(\rho) \nabla d \mathscr{W}_{t}
$$

where $\rho$ is the parameter of the invariant measure associated to the density, $\Delta$ denotes the Laplacian, and where $\sigma(\rho) \nabla d \mathscr{W}_{t}$ is an informal notation for Gaussian white noise with variance $\sigma^{2}(\rho) \int(\nabla \varphi)^{2} d x$.

In reversible interacting particle systems with (self-)duality, $\eta_{0}$ is a self-duality function, and therefore the drift term in the equation for the density field is already microscopically (i.e., without rescaling) a (linear) function of the density field. As a consequence, closing the equation and proving convergence to the limiting OrnsteinUhlenbeck process, is, for self-dual systems, particularly simple and do not require the use of a Boltzmann-Gibbs principle. This simplification suggests that, in that context, we can obtain more detailed results about fluctuation fields of more general observables. Orthogonal polynomial duality is a useful tool in the study of fluctuation fields, and associated Boltzmann-Gibbs principles, as we have seen in [3].

The density fluctuation field can be viewed as the lowest (i.e., first) order of a sequence of fields associated to orthogonal polynomials. Indeed, in all the models with orthogonal polynomial self-duality, the function $\left(\eta_{x}-\rho\right)$ is the first-order orthogonal polynomial up to a multiplicative constant. Orthogonal polynomials are indexed by finite-particle configurations, i.e., the dual configurations. If we denote by $D\left(x_{1}, \ldots x_{k} ; \eta\right)$ the orthogonal polynomial associated to the dual configuration $\sum_{i=1}^{n} \delta_{x_{i}}$, then a natural field generalizing the density fluctuation field is

$$
\mathscr{X}^{(n, k)}(\Phi, \eta)=n^{-k d / 2} \sum_{x_{i} \in \mathbb{Z}^{d}} D\left(x_{1}, \ldots, x_{k} ; \eta\right) \cdot \Phi\left(\frac{x_{1}}{n}, \ldots, \frac{x_{k}}{n}\right) .
$$

In the context of exclusion processes the case $k=2$ (orthogonal polynomial of order 2) has been studied in [11], where this field, called the quadratic fluctuation field, is shown to converge, in the limit $n \rightarrow \infty$, to the solution of a martingale problem. The quadratic variation of this 2nd-order field is proven to be a function of the 1st order field (the density field). From the result on the quadratic $(k=2)$ field one can conjecture the existence of a more general structure where the $k$ th-order orthogonal polynomials field satisfies, in the scaling limit, a martingale problem with quadratic variation depending on the $k$-1-order field.

In this paper we show exactly the emergence of a scenario of this type: within a general class of models with orthogonal polynomial self-duality we consider the fluctuation fields associated to orthogonal polynomials and prove that they converge, in the scaling limit, to the solution of a recursive system of martingale problems. We believe that this can also be a first step in the direction of defining non-linear fields, such as the square of the density field, via approximation of the identity, i.e., via a singular linear observable (cf. [11]) of the field constructed in our paper.

The rest of our paper is organized as follows. In Section 2 we define the basic models, and introduce orthogonal polynomial duality. In Section 3 we define the fluctuation fields, in Section 4 we introduce a coordinate version of the dual process, a technical tool that 
will prove to be useful later on. In Section 5 we state the main result, Theorem 5.2 below, and outline a strategy of its inductive proof. Finally, the rest of the sections are devoted to the proof of Theorem 5.2.

\section{The models}

\subsection{The infinite configuration process}

We consider an interacting particle system where an infinite number of particles randomly hop on the lattice $\mathbb{Z}^{d}$. Configurations are denoted by $\eta, \xi, \zeta$ and are elements of $\Omega \subseteq \mathbb{N}^{d}$ (where $\mathbb{N}$ denotes the natural numbers including zero). We denote by $\eta_{x}$ the number of particles at $x$ in the configuration $\eta \in \Omega$. We have in mind symmetric processes of the type independent random walkers, inclusion or exclusion. We fix two parameters $(\sigma, \alpha) \in\{0,1\} \times[0, \infty) \cup\{-1\} \times \mathbb{N}$ and we define the generator working on local functions $f: \Omega \rightarrow \mathbb{R}$ as

$$
\mathscr{L} f(\eta)=\sum_{i \in \mathbb{Z}^{d}} \sum_{r \in \mathbb{Z}^{d}} p(r) \eta_{i}\left(\alpha+\sigma \eta_{i+r}\right)\left(f\left(\eta^{i, i+r}\right)-f(\eta)\right)
$$

where $\eta^{i, i+r}$ denotes the configuration obtained from $\eta$ by removing a particle from $i$ and putting it at $i+r$. The state space $\Omega$ has to be defined and its form depends on the choice of the parameters $\alpha$ and $\sigma$.

We assume that $p(r)$ is a symmetric, finite-range, irreducible Markov transition function on $\mathbb{Z}^{d}$ :

1. Symmetry. The function $p: \mathbb{R}^{d} \rightarrow[0, \infty)$ is of the form:

$$
p\left(r_{1}, \ldots, r_{d}\right)=p\left(\left|r_{1}\right|, \ldots,\left|r_{d}\right|\right)
$$

and such that $p\left(\mathbf{r}_{\sigma}\right):=p\left(r_{\sigma(1)}, \ldots, r_{\sigma(d)}\right)=p\left(r_{1}, \ldots, r_{d}\right)$ for all $\sigma \in \mathscr{P}(d)$, the set of permutations of $\{1, \ldots, d\}$.

2. Finite-range. There exists a finite subset $\mathscr{R} \subset \mathbb{Z}^{d}$ of the form $\mathscr{R}=[-R, R]^{d} \cap \mathbb{Z}^{d}$, for some $R \in \mathbb{N}, R>1$, such that $p(r)=0$ for all $r \notin \mathscr{R}$.

3. Irreducibility. For all $x, y \in \mathbb{Z}^{d}$ there exists a sequence $i_{1}=x, \ldots, i_{n}=y$ such that

$$
\prod_{k=1}^{n-1} p\left(i_{k}-i_{k+1}\right)>0
$$

We will also assume, without loss of generality, that $p(0)=0$, and denote by $\chi$ the second moment:

$$
\chi:=\sum_{r \in \mathscr{R}} r_{\ell}^{2} \cdot p(r), \quad \text { for all } \ell \in\{1, \ldots, d\} .
$$

Remark 2.1. The symmetry assumption (2.2) is crucial in order to be able to have and apply orthogonal self-duality.

For the associated Markov processes on $\Omega$, we use the notation $\{\eta(t): t \geq 0\}, \eta_{x}(t)$ denoting the number of particles at time $t$ at location $x \in \mathbb{Z}^{d}$. These particle systems have a one-parameter family of homogeneous (w.r.t. translations) reversible and ergodic product measures $\nu_{\rho}, \rho>0$, indexed by the density of particles, i.e.,

$$
\int \eta_{0} d \nu_{\rho}=\rho
$$

The nature of the underlying dynamics and the type of reversible measure we obtain is regulated by the parameter $\sigma \in \mathbb{Z}$ as follows. 
Independent random walkers (IRW): This particle system corresponds to the choice $\sigma=0$ and the intensity parameter $\alpha \in \mathbb{R}$ regulates the rate at which the walkers move. The reversible measures $\nu_{\rho}, \rho>0$ are products of Poisson distributions with parameter $\rho, \nu_{\rho}=\otimes_{i \in \mathbb{Z}^{d}} \operatorname{Pois}(\rho)$, i.e. the marginals are given by

$$
\mathbb{P}_{\nu_{\rho}}\left(\eta_{i}=n\right)=\frac{1}{Z_{\rho}} \cdot \frac{\rho^{n}}{n !}, \quad Z_{\rho}=e^{-\rho}, \quad \forall i \in \mathbb{Z}^{d} .
$$

Symmetric exclusion process $(\operatorname{SEP}(\alpha))$ : The choice $\sigma=-1$ results in exclusion interaction. For this process the parameter $\alpha$ takes values in the set of natural numbers $\mathbb{N}$, as it determines the maximum number of particles allowed per site. This system is well known to have reversible measures $\nu_{\rho}, \rho \in(0, \alpha)$, that are products of Binomial distributions: $\nu_{\rho}=\otimes_{i \in \mathbb{Z}^{d}} \operatorname{Binom}\left(\alpha, \frac{\rho}{\alpha}\right)$ whose marginals are given by

$$
\mathbb{P}_{\nu_{\rho}}\left(\eta_{i}=n\right)=\frac{1}{Z_{\alpha, \rho}} \cdot\left(\begin{array}{l}
\alpha \\
n
\end{array}\right) \cdot\left(\frac{\rho}{\alpha-\rho}\right)^{n}, \quad Z_{\alpha, \rho}=\left(\frac{\alpha}{\alpha-\rho}\right)^{\alpha}, \quad \forall i \in \mathbb{Z}^{d} .
$$

Symmetric inclusion process $(\operatorname{SIP}(\alpha))$ : The choice $\sigma=1$ gives rise to an interaction of inclusion-type consisting of particles attracting each other. The SIP is known to have products of Negative-Binomial distributions as reversible measures, i.e. $\nu_{\rho}$, $\rho>0$ with $\nu_{\rho}=\otimes_{i \in \mathbb{Z}^{d}} \operatorname{Neg}$-Binom $\left(\alpha, \frac{\rho}{\rho+\alpha}\right)$ with marginals

$$
\mathbb{P}_{\nu_{\rho}}\left(\eta_{i}=n\right)=\frac{1}{Z_{\alpha, \rho}} \cdot \frac{\Gamma(\alpha+n)}{\Gamma(\alpha) \cdot n !}\left(\frac{\rho}{\alpha+\rho}\right)^{n}, \quad Z_{\alpha, \rho}=\left(\frac{\alpha+\rho}{\alpha}\right)^{\alpha}, \quad \forall i \in \mathbb{Z}^{d} .
$$

Remark 2.2. Notice that for the three processes we have that all moments are finite.

The definition of the state space $\Omega$ is different in each case, depending on whether there are restrictions or not on the total number of particles allowed per site. This is finite for the exclusion process, thus, for $\operatorname{SEP}(\alpha)$, we have $\Omega=\{0,1, \ldots, \alpha\}^{\mathbb{Z}^{d}}$. The situation is different in the cases of IRW and SIP, for which, in principle, there are no restrictions. Nevertheless, one has to avoid explosions of the number of particles in a given site. For this reason the characterization of $\Omega$ in these cases (i.e. for $\sigma \geq 0$ ) is a more subtle problem whose treatment is beyond the scope of this thesis. Here we will restrict ourselves by implicitly defining $\Omega$ as the set of configurations in $\mathbb{N}^{\mathbb{Z}^{d}}$ whose evolution $\eta(t)$ is well-defined and belonging to $\Omega$ for all subsequent times $t \geq 0$. We refer the reader to [1] and [7] for examples on conditions sufficient to guarantee the well-definedness. A possible such subset is the set of tempered configurations. This is the set of configurations $\eta$ such that there exist $C, \beta \in \mathbb{R}$ that satisfy $|\eta(x)| \leq C|x|^{\beta}$ for all $x \in \mathbb{R}$. From this set of configurations, and via a finite volume approximation using self-duality one can follow the lines of [1] and [7] to show the well-definedness.

\subsection{The finite configuration processes}

The process introduced in Section 2.1 can also be realized with a fixed finite number of particles. For a process with $k \in \mathbb{N}$ particles we denote by $\Omega_{k}$ its state space, more precisely:

$$
\Omega_{k}=\left\{\xi \in \Omega:\|\xi\|:=\sum_{x \in \mathbb{Z}^{d}} \xi_{x}=k\right\} .
$$

We will then denote by $\{\xi(t): t \geq 0\}$ the $\Omega_{k}$-valued Markov process, with infinitesimal generator given by

$$
\mathscr{L}^{(k)} f(\xi)=\sum_{i \in \mathbb{Z}^{d}} \sum_{r \in \mathscr{R}} p(r) \xi_{i}\left(\alpha+\sigma \xi_{i+r}\right)\left(f\left(\xi^{i, i+r}\right)-f(\xi)\right)
$$

working on functions $f: \Omega_{k} \rightarrow \mathbb{R}$. 
Remark 2.3. Notice that from the fact that any configuration $\xi \in \Omega_{k}$ has exactly $k$ particles, there is no room for explosions (meaning infinitely many particles coexisting on one site). Therefore the process $\{\xi(t): t \geq 0\}$ is well-defined.

We now define the following inner product for functions $f, g: \Omega_{k} \rightarrow \mathbb{R}$ :

$$
\langle f, g\rangle_{\Lambda}=\sum_{\xi \in \Omega_{k}} f(\xi) g(\xi) \Lambda(\xi)
$$

where the weight $\Lambda: \Omega_{k} \rightarrow \mathbb{R}$, which does not depend on $k$, is given by:

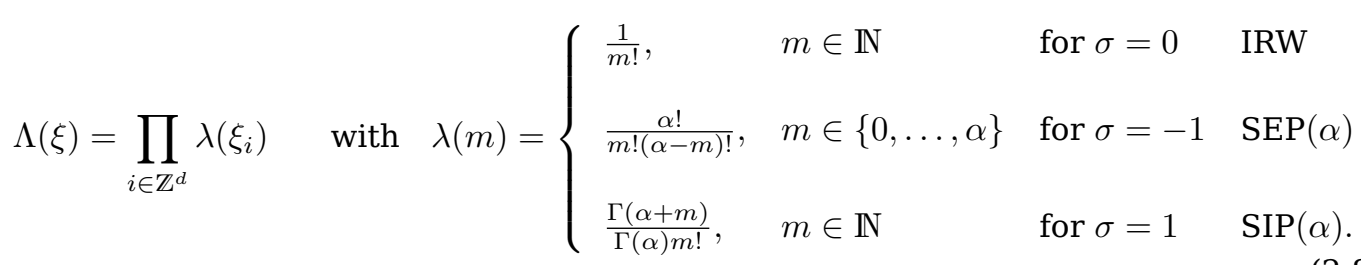

The weight $\Lambda(\cdot)$ satisfies detailed balance and as a consequence of this we can infer that the $k$-particles generator $\mathscr{L}^{(k)}$ is self-adjoint with respect to the inner product $\langle\cdot, \cdot\rangle_{\Lambda}$, i.e. for all $f, g \in L^{2}\left(\Omega_{k}, \Lambda\right)$ we have

$$
\left\langle f, \mathscr{L}^{(k)} g\right\rangle_{\Lambda}=\left\langle\mathscr{L}^{(k)} f, g\right\rangle_{\Lambda}
$$

Therefore $\Lambda(\cdot)$ can be thought of as a reversible $\sigma$-finite measure on each $\Omega_{k}$.

\subsection{Orthogonal polynomial self-duality}

The processes defined in Section 2.1 share a self-duality property that will be crucial in our analysis. Define the set

$$
\Omega_{f}=\bigcup_{k \in \mathbb{N}} \Omega_{k}
$$

of configurations with a finite number of particles, the self-duality functions that we consider in this paper are functions $D_{\rho}: \Omega_{f} \times \Omega \rightarrow \mathbb{R}$ parametrized by the density $\rho>0$ satisfying the following properties.

1. Self-duality:

$$
\mathbb{E}_{\eta}\left[D_{\rho}(\xi, \eta(t))\right]=\mathbb{E}_{\xi}\left[D_{\rho}(\xi(t), \eta)\right] \quad \text { for all } \quad \xi \in \Omega_{f}, \eta \in \Omega
$$

or, equivalently,

$$
\left[\mathscr{L} D_{\rho}(\xi, \cdot)\right](\eta)=\left[\mathscr{L}^{(k)} D_{\rho}(\cdot, \eta)\right](\xi) \quad \text { for all } \quad \xi \in \Omega_{f}, \eta \in \Omega \text {. }
$$

2. Factorized polynomials:

$$
D_{\rho}(\xi, \eta)=\prod_{i \in \mathbb{Z}^{d}} d_{\rho}\left(\xi_{i}, \eta_{i}\right)
$$

where $d_{\rho}(0, n)=1$, and $d_{\rho}(k, \cdot)$ is a polynomial of degree $k$.

3. Orthogonality:

$$
\int D_{\rho}(\xi, \eta) D_{\rho}\left(\xi^{\prime}, \eta\right) d \nu_{\rho}(\eta)=\delta_{\xi, \xi^{\prime}} \cdot \frac{1}{\mu_{\rho}(\xi)}
$$

where

$$
\mu_{\rho}(\xi):=\left(\int D_{\rho}(\xi, \eta)^{2} d \nu_{\rho}(\eta)\right)^{-1}
$$


Remark 2.4. Notice that, as a consequence of the orthogonality property (2.13), we have that

$$
\int \mathbb{E}_{\eta}\left[D_{\rho}(\xi, \eta(t))\right] \cdot D_{\rho}\left(\xi^{\prime}, \eta\right) d \nu_{\rho}(\eta)=p_{t}\left(\xi, \xi^{\prime}\right) \cdot \frac{1}{\mu_{\rho}\left(\xi^{\prime}\right)}
$$

where $p_{t}(\cdot, \cdot)$ is the transition probability function of the dual process $\{\xi(t): t \geq 0\}$. Moreover, if we use the reversibility of the measure $\nu_{\rho}$ on the LHS of (2.15) we obtain

$$
\begin{aligned}
p_{t}\left(\xi, \xi^{\prime}\right) \cdot \frac{1}{\mu_{\rho}\left(\xi^{\prime}\right)} & =\int \mathbb{E}_{\eta}\left[D_{\rho}(\xi, \eta(t))\right] \cdot D_{\rho}\left(\xi^{\prime}, \eta\right) d \nu_{\rho}(\eta) \\
& =\int D_{\rho}(\xi, \eta) \cdot \mathbb{E}_{\eta}\left[D_{\rho}\left(\xi^{\prime}, \eta(t)\right)\right] d \nu_{\rho}(\eta) \\
& =p_{t}\left(\xi^{\prime}, \xi\right) \cdot \frac{1}{\mu_{\rho}(\xi)}
\end{aligned}
$$

which, by detailed balance, implies the reversibility of the measure $\mu_{\rho}(\xi)$. This in turn implies that there exists a constant $c(k, \rho)$ such that

$$
\Lambda(\xi)=c(k, \rho) \cdot \mu_{\rho}(\xi) \quad \text { for all } \xi \in \Omega_{k} .
$$

Remark 2.5. Notice that by Remark 2.2 we have that $\mu_{\rho}(\xi)<\infty$ for every $\xi \in \Omega_{f}$. Moreover, the measure $\mu_{\rho}$ is not a probability measure.

From now on we will often suppress the dependence on the parameter $\rho$, of the duality functions $D(\cdot, \cdot)=D_{\rho}(\cdot, \cdot)$, in order not to overload the notation. The same omission will be done for the single site duality-polynomials $d(\cdot, \cdot)$, and any other orthogonal polynomial introduced below.

For each of the processes we are considering, the orthogonal duality polynomials are given as follows.

IRW: Charlier polynomials. The duality polynomials are given by

$$
d(m, n)=C(m, n)
$$

where $C(m, \cdot)$ is the Charlier polynomial of degree $m$ that we characterize by means of the following generating function:

$$
\sum_{m=0}^{\infty} C(m, n) \cdot \frac{t^{m}}{m !}=e^{-t}\left(\frac{\rho+t}{\rho}\right)^{n} .
$$

We can differentiate the RHS of (2.18) with respect to $t$, and evaluate at $t=0$, to obtain that the first three Charlier (and self-duality) polynomials are:

$$
\begin{aligned}
& d(0, n)=C(0, n)=1 \\
& d(1, n)=C(1, n)=\frac{1}{\rho}(n-\rho), \\
& d(2, n)=C(2, n)=\frac{1}{\rho^{2}}\left(n(n-1)-2 \rho n+\rho^{2}\right) .
\end{aligned}
$$

$\operatorname{SEP}(\alpha)$ : Krawtchouk polynomials. For the SEP the duality polynomials are given by

$$
d(m, n)=\frac{m !(\alpha-m) !}{\alpha !} \cdot K(m, n)
$$

where $K(m, \cdot)$ is the Krawtchouk polynomial of degree $m$ whose generating function is

$$
\sum_{m=0}^{\infty} K(m, n) \cdot t^{m}=(1-t)^{\alpha}\left(\frac{1+\left(\frac{\alpha-\rho}{\rho}\right) t}{1-t}\right)^{n} .
$$


With analogous computations to the IRW case, the first Krawtchouk polynomials are:

$$
\begin{aligned}
K(0, n) & =1 \\
K(1, n) & =\frac{\alpha}{\rho}(n-\rho) \\
K(2, n) & =\left(\frac{\alpha}{\rho}\right)^{2} n(n-1)-2\left(\frac{\alpha}{\rho}\right)(\alpha-1) n+\alpha(\alpha-1),
\end{aligned}
$$

with corresponding single-site duality polynomials:

$$
\begin{aligned}
& d(0, n)=1 \\
& d(1, n)=\frac{1}{\rho}(n-\rho) \\
& d(2, n)=\frac{2 \alpha}{\rho^{2}(\alpha-1)}\left(n(n-1)-\frac{2 \rho(\alpha-1)}{\alpha} n+\frac{\rho^{2}(\alpha-1)}{\alpha}\right) .
\end{aligned}
$$

Remark 2.6. Notice that these polynomials are only defined for $m, n \leq \alpha$.

SIP $(\alpha)$ : Meixner polynomials. In this case the polynomials satisfying the self-duality relation are given by the following normalization of the Meixner polynomials

$$
d(m, n)=\frac{\Gamma(\alpha)}{\Gamma(\alpha+m)} \cdot M(m, n)
$$

where $M(m, \cdot)$ is the Meixner polynomial of degree $m$ with generating function

$$
\sum_{m=0}^{\infty} M(m, n) \cdot \frac{t^{m}}{m !}=(1-t)^{-\alpha}\left(\frac{1-\frac{(\alpha+\rho) t}{\rho}}{1-t}\right)^{n} .
$$

The first Meixner polynomials are:

$$
\begin{aligned}
& M(0, n)=1 \\
& M(1, n)=-\frac{\alpha}{\rho}(n-\rho), \\
& M(2, n)=\left(\frac{\alpha}{\rho}\right)^{2} n(n-1)-2\left(\frac{\alpha}{\rho}\right)(\alpha+1) n+\alpha(\alpha+1) .
\end{aligned}
$$

with corresponding single-site duality polynomials are:

$$
\begin{aligned}
& d(0, n)=1 \\
& d(1, n)=-\frac{1}{\rho}(n-\rho) \\
& d(2, n)=\frac{\alpha}{\rho^{2}(\alpha+1)}\left(n(n-1)-\frac{2 \rho(\alpha+1)}{\alpha} n+\frac{\rho^{2}(\alpha+1)}{\alpha}\right) .
\end{aligned}
$$

We refer the reader to [13] and [5] for more details on these polynomials and their generating functions. For proofs of self-duality with these orthogonal polynomials we refer to [10] and [14].

\section{Fluctuation fields}

The density fluctuation field $\mathscr{X}$ is the stochastic object usually defined to study fluctuations of density around its expected limit. This field corresponds to a central limit type of rescaling of the density field, i.e.

$$
\mathscr{X}_{t}^{(n)}(\varphi, \eta):=n^{-d / 2} \sum_{x \in \mathbb{Z}^{d}} \varphi(x / n)\left(\eta_{x}\left(n^{2} t\right)-\rho\right) .
$$


where $\varphi$ is an element of the Schwartz space $S\left(\mathbb{R}^{d}\right)$, i.e., the space of all smooth functions whose derivatives are rapidly decreasing:

$$
S\left(\mathbb{R}^{d}\right)=\left\{\varphi \in C^{\infty}\left(\mathbb{R}^{d}\right): \sup _{x \in \mathbb{R}^{d}}\left|x^{\alpha} D^{\beta} \varphi\right|<\infty, \forall \alpha, \beta \in \mathbb{N}\right\}
$$

where $C^{\infty}\left(\mathbb{R}^{d}\right)$ is the space of smooth functions.

Fields of this type have been intensively studied in the literature. For different models, see for example [12] for the case of the ZRP, the sequence $\mathscr{X}_{t}^{(n)}$ is proven to converge to a limiting field $\mathscr{X}_{t}$ that is identified as the distribution-valued random variable satisfying the following martingale problem: for any $\varphi \in S\left(\mathbb{R}^{d}\right)$ the process

$$
M_{t}(\varphi)=\mathscr{X}_{t}(\varphi)-\mathscr{X}_{0}(\varphi)-\frac{\chi \alpha}{2} \int_{0}^{t} \mathscr{X}_{s}(\Delta \varphi) d s
$$

is a square integrable continuous martingale of quadratic variation given by the expression:

$$
\chi \rho(\alpha+\sigma \rho)\|\nabla \varphi(x)\|^{2} \cdot t .
$$

Remark 3.1. The factors $\frac{\chi \alpha}{2}$ and $\chi \rho(\alpha+\sigma \rho)$ come from the symmetry assumption (2.2). We refer to Section 6.1 for more details on how these factors emerge on the fields of order $k$. Under the absence of (2.2) we cannot guarantee obtaining the same multiplicative factors.

Following a procedure analogous to the one given in Chapter 11, pages 290-291, of [12], the martingale problem (3.3)-(3.4) can be rewritten as:

$$
\mathscr{X}_{t}(\varphi)=\mathscr{X}_{0}(\varphi)+\frac{\chi \alpha}{2} \int_{0}^{t} \mathscr{X}_{s}(\Delta \varphi) d s+\sqrt{\chi \rho(\alpha+\sigma \rho)}\|\nabla \varphi(x)\| \mathscr{W}_{t}(\varphi)
$$

where $\mathscr{W}_{t}$ is a generalized Brownian motion with covariance

$$
\operatorname{cov}\left[\mathscr{W}_{t}(\varphi), \mathscr{W}_{s}(\psi)\right]=\min (t, s) \int_{\mathbb{R}} \frac{\nabla \varphi(x)}{\|\nabla \varphi(x)\|} \frac{\nabla \psi(x)}{\|\nabla \psi(x)\|} d x .
$$

Formally speaking, (3.5) is equivalent to say that the limiting field $\mathscr{X}_{t}$ satisfies (cf. [12], pages 290-291) the Ornstein-Uhlenbeck equation:

$$
d \mathscr{X}_{t}=\frac{\chi \alpha}{2} \Delta \mathscr{X}_{t} d t+\sqrt{\chi \rho(\alpha+\sigma \rho)} \nabla d \mathscr{W}_{t},
$$

Here $\nabla d \mathscr{W}_{t}$ has to be interpreted by saying that the integral

$$
\int_{0}^{t} \nabla d \mathscr{W}_{s}(\varphi)
$$

is a continuous martingale of quadratic variation:

$$
t \cdot\|\nabla \varphi(x)\| .
$$

We refer the reader to [6] for a precise statement on the convergence for the case of the exclusion process, corresponding, in our setting, to the case $\alpha=1$ and $\sigma=-1$.

The density field (3.1) can be written, in our context, in terms of our orthogonal polynomial dualities $D_{\rho}(\xi, \eta)$ by choosing $\xi \in \Omega_{1}$. Indeed, in all models considered we have that there exists a constant $c_{\sigma, \alpha, \rho}$ such that

$$
D_{\rho}\left(\delta_{x}, \eta\right)=c_{\sigma, \alpha, \rho}\left(\eta_{x}-\rho\right)
$$


where

$$
c_{\sigma, \alpha, \rho}=\left\{\begin{array}{lll}
1 / \rho & \text { if } & \sigma=0 \\
-1 / \rho & \text { if } & \sigma=1 \\
1 / \rho & \text { if } & \sigma=-1
\end{array} .\right.
$$

Later on, in order not to overload notation we will suppress the dependence on $\rho$ and $\alpha$ and just write $c_{\sigma}$. From (3.10) we observe that the field (3.1) can be rewritten (modulo a multiplicative constant) as

$$
\mathscr{X}_{t}^{(n, 1)}(\varphi)=n^{-d / 2} \sum_{x \in \mathbb{Z}^{d}} \varphi\left(\frac{x}{n}\right) D_{\rho}\left(\delta_{x}, \eta\left(n^{2} t\right)\right)
$$

where the superindex $(n, 1)$ suggests that, in some sense, this is the first-order density field. Using (3.10) and (3.7) the formal limiting SPDE for $\mathscr{X}_{t}$ is

$$
d \mathscr{X}_{t}=\frac{\chi \alpha}{2} \Delta \mathscr{X}_{t} d t+c_{\sigma} \sqrt{\chi \rho(\alpha+\sigma \rho)} \nabla d \mathscr{W}_{t}
$$

The observation that the field (3.1) can be expressed in terms of duality polynomials opens the possibility of defining higher-order fields and study their scaling limits. For $k \in \mathbb{N}, k \geq 1$ we define the $k$-th order field as

$$
\begin{aligned}
\mathscr{X}^{(n, k)}\left(\varphi^{(k)}, \eta\right):=\mathscr{Y}^{(n, k)}(\Phi, \eta) & :=n^{-k d / 2} \sum_{\xi \in \Omega_{k}}\left(\prod_{x \in \mathbb{Z}^{d}} \varphi\left(\frac{x}{n}\right)^{\xi_{x}}\right) \Lambda(\xi) \cdot D_{\rho}(\xi, \eta) \\
& =n^{-k d / 2} \sum_{\xi \in \Omega_{k}}\left(\prod_{x \in \mathbb{Z}^{d}} \varphi\left(\frac{x}{n}\right)^{\xi_{x}} \cdot \lambda\left(\xi_{x}\right) \cdot d_{\rho}\left(\xi_{x}, \eta_{x}\right)\right)
\end{aligned}
$$

where $\varphi \in S\left(\mathbb{R}^{d}\right)$ is a test function, $\Lambda$ is as in (2.8), and

$$
\begin{gathered}
\varphi^{(k)}:=\bigotimes_{i=1}^{k} \varphi \\
\Phi(\xi)=\prod_{x \in \mathbb{Z}^{d}} \varphi(x)^{\xi_{x}}, \quad \Phi_{n}(\xi)=\prod_{x \in \mathbb{Z}^{d}} \varphi\left(\frac{x}{n}\right)^{\xi_{x}}
\end{gathered}
$$

In the rest of this work, we will refer to test functions of the type $\varphi^{(k)}$ as symmetric elements of the Schwartz space $S\left(\mathbb{R}^{k d}\right)$. Likewise, the functions $\Phi: \Omega_{k} \rightarrow \mathbb{R}$, given by (3.16), will be considered as elements of the Schwartz space of test functions over configuration space.

Notice that there is no difference between $\mathscr{X}^{(n, k)}\left(\varphi^{(k)}, \eta\right)$ and $\mathscr{Y}^{(n, k)}(\Phi, \eta)$ besides that the latter works on test functions over configuration space, i.e., $\Phi \in S\left(\Omega_{k}\right)$, while the former works on test functions $\varphi^{(k)} \in S\left(\mathbb{R}^{k d}\right)$. Then, using the notation

$$
\begin{gathered}
\mathfrak{D}_{\rho}(\xi, \eta):=\Lambda(\xi) \cdot D_{\rho}(\xi, \eta), \quad \mathfrak{d}_{\rho}(m, n)=\lambda(m) \cdot d_{\rho}(m, n) \\
\mathfrak{D}_{\rho}(\xi, \eta)=\prod_{i \in \mathbb{Z}^{d}} \mathfrak{d}_{\rho}\left(\xi_{i}, \eta_{i}\right)
\end{gathered}
$$

we can rewrite the $k$-th order field (3.14) as

$$
\mathscr{Y}^{(n, k)}(\Phi, \eta):=n^{-k d / 2} \sum_{\xi \in \Omega_{k}} \Phi_{n}(\xi) \cdot \mathfrak{D}_{\rho}(\xi, \eta)
$$

and define:

$$
\mathscr{Y}_{t}^{(n, k)}(\Phi):=\mathscr{Y}^{(n, k)}\left(\Phi, \eta\left(n^{2} t\right)\right)
$$

The choice of multiplying the duality function by the measure $\Lambda(\cdot)$ in (3.17) is dictated simply by computational convenience that, even if obscure at the moment, will be made clearer in the course of this work. 


\section{First example: second-order fluctuation fields for the $\operatorname{SEP}(1)$}

Let us specialize these fields to the case of the one-dimensional symmetric exclusion process for $k=2$. This means that we are taking:

$$
d=1, \quad \alpha=1, \text { and } \quad \sigma=-1 \text {. }
$$

In this case we have:

$$
\begin{aligned}
\mathscr{X}^{(n, 2)}\left(\varphi^{(2)}, \eta\right) & =\frac{1}{n} \sum_{\xi \in \Omega_{2}}\left(\prod_{x \in \mathbb{Z}} \varphi\left(\frac{x}{n}\right)^{\xi_{x}}\right) \Lambda(\xi) \cdot D_{\rho}(\xi, \eta) \\
& =\frac{1}{2 n} \sum_{\substack{x, y \in \mathbb{Z} \\
x \neq y}} \varphi\left(\frac{x}{n}\right) \varphi\left(\frac{y}{n}\right) \Lambda\left(\delta_{x}+\delta_{y}\right) D_{\rho}\left(\delta_{x}+\delta_{y}, \eta\right) \\
& =\frac{1}{2 \rho^{2}}\left[\frac{1}{n} \sum_{\substack{x, y \in \mathbb{Z} \\
x \neq y}} \varphi\left(\frac{x}{n}\right) \varphi\left(\frac{y}{n}\right)\left(\eta_{x}-\rho\right)\left(\eta_{y}-\rho\right)\right]
\end{aligned}
$$

where in the second line, in order to get rid of the sum at the diagonal, we used the fact that for $\operatorname{SEP}(1)$ we have $D_{\rho}\left(2 \delta_{x}, \eta\right)=0$. Notice that in the last line we used (2.8) and (2.22).

Remark 3.2. Notice that the previous field corresponds, modulo a multiplicative factor, to the quadratic field introduced earlier in [11]. Also notice that the previous field is not the same as the quadratic field introduced in [2].

\section{Second example: second-order fluctuation fields for IRW $(1)$}

Let us now look at the case of one-dimensional independent random walkers. This means that we are taking:

$$
d=1, \quad \alpha=1, \text { and } \quad \sigma=0
$$

Analogous to the case of $\operatorname{SEP}(1)$, in this case we have:

$$
\begin{aligned}
\mathscr{X}^{(n, 2)}\left(\varphi^{(2)}, \eta\right) & =\frac{1}{n} \sum_{\xi \in \Omega_{2}}\left(\prod_{x \in \mathbb{Z}} \varphi\left(\frac{x}{n}\right)^{\xi_{x}}\right) \Lambda(\xi) \cdot D_{\rho}(\xi, \eta) \\
& =\frac{1}{2 n} \sum_{\substack{x, y \in \mathbb{Z} \\
x \neq y}} \varphi\left(\frac{x}{n}\right) \varphi\left(\frac{y}{n}\right) \Lambda\left(\delta_{x}+\delta_{y}\right) D_{\rho}\left(\delta_{x}+\delta_{y}, \eta\right) \\
& +\frac{1}{n} \sum_{x \in \mathbb{Z}} \varphi\left(\frac{x}{n}\right)^{2} \Lambda\left(2 \delta_{x}\right) D_{\rho}\left(2 \delta_{x}, \eta\right) \\
& =\frac{1}{2 n \rho^{2}} \sum_{\substack{x, y \in \mathbb{Z} \\
x \neq y}} \varphi\left(\frac{x}{n}\right) \varphi\left(\frac{y}{n}\right)\left(\eta_{x}-\rho\right)\left(\eta_{y}-\rho\right) \\
& +\frac{1}{2 n \rho^{2}} \sum_{x \in \mathbb{Z}} \varphi\left(\frac{x}{n}\right)^{2}\left(\eta_{x}\left(\eta_{x}-1\right)-2 \rho \eta_{x}+\rho^{2}\right)
\end{aligned}
$$

Remark 3.3. Notice that different to the case of $\operatorname{SEP}(1)$, in this case we have that the second-order duality polynomials do not vanish and as a consequence we have a contribution coming from the diagonal (i.e., the second summation in the RHS of (3.24)). 


\section{The coordinate process}

The coordinate process can be described in words as the process obtained by labeling the particles of a process with a finite number of particles. Thinking of $k \in \mathbb{N}$ as the number of particles, we want to introduce a family of permutation-invariant coordinate processes $\left\{X^{(k)}(t): t \geq 0\right\}$ compatible with the finite configuration processes $\{\xi(t): t \geq$ $0\}$ on $\Omega_{k}$. Here the coordinate process is a Markov process on $\mathbb{Z}^{d k}$ with

$$
X^{(k)}(t)=\left(X_{1}(t), \ldots, X_{k}(t)\right), \quad X_{i}(t) \in \mathbb{Z}^{d}, \quad \forall i=1, \ldots, k
$$

$X_{i}(t)$ being the position of the $i$-th particle at time $t \geq 0$. For a further explanation of the notion of compatibility we refer the reader to [4].

Denote by $\mathbf{x} \in \mathbb{Z}^{k d}$ the coordinate vector $\mathbf{x}:=\left(x_{1}, \ldots, x_{k}\right)$, with $x_{i} \in \mathbb{Z}^{d}$, for $i=$ $1, \ldots, k$. The coordinate process $\left\{X^{(k)}(t): t \geq 0\right\}$ is defined by means of its infinitesimal generator:

$$
L^{(k)} f(\mathbf{x})=\sum_{i=1}^{k} \sum_{r \in \mathscr{R}} p(r)\left(\alpha+\sigma \sum_{\substack{j=1 \\ j \neq i}}^{k} \mathbf{1}_{x_{j}=x_{i}+r}\right)\left(f\left(\mathbf{x}^{i, i+r}\right)-f(\mathbf{x})\right)
$$

where $\mathbf{x}^{i, i+r}$ denotes $\mathbf{x}$ after moving the particle in position $x_{i}$ to position $x_{i}+r \in \mathbb{Z}^{d}$.

Notice that for $\mathbf{x} \in \mathbb{Z}^{k d}$ the compatible configuration $\xi(\mathbf{x}) \in \Omega_{k}$ is given by

$$
\xi(\mathbf{x})=\left(\xi_{i}(\mathbf{x}), i \in \mathbb{Z}^{d}\right) \quad \text { with } \quad \xi_{i}(\mathbf{x})=\sum_{j=1}^{k} \mathbf{1}_{x_{j}=i}
$$

\subsection{Product $\sigma$-finite reversible measures}

It is possible to verify, by means of detailed balance, that the coordinate-process $\left\{X^{(k)}(t): t \geq 0\right\}$ admits a reversible $\sigma$-finite measure that is given by

$$
\Pi(\mathbf{x})=\frac{\Lambda(\xi(\mathbf{x}))}{N(\xi(\mathbf{x}))}=\frac{1}{k !} \prod_{i \in \mathbb{Z}^{d}} \xi_{i}(\mathbf{x}) ! \cdot \lambda\left(\xi_{i}(\mathbf{x})\right) \quad \text { for } \quad \mathbf{x} \in \mathbb{Z}^{k d}
$$

where $\lambda$ is given as in (2.8), and with

$$
N(\xi):=\left|\left\{\mathbf{x} \in \mathbb{Z}^{k d}: \xi(\mathbf{x})=\xi\right\}\right|=\frac{k !}{\prod_{i \in \mathbb{Z}^{d}} \xi_{i} !}
$$

then we can rewrite $\Pi$ in the product form:

$$
\Pi(\mathbf{x})=\frac{1}{k !} \prod_{i \in \mathbb{Z}^{d}} \pi\left(\xi_{i}(\mathbf{x})\right), \quad \mathbf{x}=\left(x_{1}, \ldots, x_{k}\right) \in \mathbb{Z}^{k d}
$$

with $\pi$ given as follows:

$$
\pi(m)=\lambda(m)=\left\{\begin{array}{llll}
\frac{1}{m !}, & m \in \mathbb{N} & \text { for } \sigma=0 & \text { IRW } \\
\frac{\alpha !}{(\alpha-m) ! m !}, & m \in\{0, \ldots, \alpha\} & \text { for } \sigma=-1 & \operatorname{SEP}(\alpha) \\
\frac{\Gamma(\alpha+m)}{\Gamma(\alpha) m !}, & m \in \mathbb{N} & \text { for } \sigma=1 & \operatorname{SIP}(\alpha)
\end{array}\right.
$$

Given the measures $\Pi$, we now consider the spaces of permutation-invariant functions:

$$
\hat{L}^{2}\left(\mathbb{Z}^{k d}, \Pi\right):=\left\{f \in L^{2}\left(\mathbb{Z}^{k d}, \Pi\right): f(\mathbf{x})=f\left(\mathbf{x}_{\sigma}\right), \forall \sigma \in \mathscr{P}(k)\right\}
$$


with $\mathscr{P}(k)$ denoting the set of all possible permutations of the set $\{1,2,3, \ldots k\}$. We endowed the space $\hat{L}^{2}\left(\mathbb{Z}^{k d}, \Pi\right)$ with the inner product given by:

$$
\langle f, g\rangle_{\Pi}=\sum_{\mathbf{x} \in \mathbb{Z}^{k d}} f(\mathbf{x}) g(\mathbf{x}) \Pi(\mathbf{x}) .
$$

Remark 4.1. Notice that any function $f \in \hat{L}^{2}\left(\mathbb{Z}^{k d}, \Pi\right)$ can be interpreted also as a function on the configuration space. In this work we will extensively use this fact by changing between interpretations sometimes from one line to another in the same derivation.

Remark 4.2. As a consequence of reversibility of the measures $\Pi$, we can infer that the $k$-particles generator $L^{(k)}$ is self-adjoint with respect to the inner product $\langle\cdot, \cdot\rangle_{\Pi}$, i.e.

$$
\left\langle f, L^{(k)} g\right\rangle_{\Pi}=\left\langle L^{(k)} f, g\right\rangle_{\Pi}
$$

for all $f, g \in \hat{L}^{2}\left(\mathbb{Z}^{k d}, \Pi\right)$.

\subsection{The fluctuation fields in coordinate notation}

It is possible to rewrite the fluctuation field (3.14) in the coordinate variables. Notice that in this context the test function $\Phi$ defined in (3.16) becomes a tensor function:

$$
\Phi(\xi(\mathbf{x}))=\prod_{i=1}^{k} \varphi\left(x_{i}\right)
$$

i.e. it is the homogeneous $k$-tensor test function $\varphi^{\otimes k} \in S\left(\mathbb{R}^{k d}\right)$ of the form

$$
\Phi \circ \xi=\varphi^{\otimes k}:=\bigotimes_{i=1}^{k} \varphi
$$

then, after a change of variable in the sum we can rewrite the $k$-th field as follows

$$
\mathscr{X}^{(n, k)}\left(\varphi^{(k)}, \eta\right)=\mathscr{Y}^{(n, k)}(\Phi, \eta)=n^{-k d / 2} \sum_{\mathbf{x} \in \mathbb{Z}^{k d}} \varphi^{(k)}\left(\frac{\mathbf{x}}{n}\right) \cdot \Pi(\mathbf{x}) \cdot D(\xi(\mathbf{x}), \eta) .
$$

Notice that we can also let the field $\mathscr{X}$ act on a general $f \in S\left(\mathbb{R}^{k d}\right)$ as expected, i.e.,

$$
\mathscr{X}^{(n, k)}(f, \eta)=n^{-k d / 2} \sum_{\mathbf{x} \in \mathbb{Z}^{k d}} f\left(\frac{\mathbf{x}}{n}\right) \cdot \Pi(\mathbf{x}) \cdot D(\xi(\mathbf{x}), \eta) .
$$

Remark 4.3. Because we deal with unlabeled particle systems it is natural to define the higher-order fluctuation fields acting on symmetric test functions $\Phi$ i.e. on elements of the Schwartz space $S\left(\mathbb{R}^{k d}\right)$ that are permutation-invariant: $\Phi\left(x_{\sigma(1)}, \ldots, x_{\sigma(k)}\right)=$ $\Phi\left(x_{1}, \ldots, x_{k}\right)$ for all $\sigma \in \mathscr{P}(k)$, the set of permutations of $\{1, \ldots, k\}$.

Remark 4.4. The set of test functions of the form $\varphi^{\otimes k}$ is dense in the space of symmetric Schwartz test functions. This can be seen in two steps. First, linear combination of tensors are dense in $S\left(\mathbb{R}^{k d}\right)$. Second, restricting to symmetric linear combinations of elements in $S\left(\mathbb{R}^{k d}\right)$, we have that by polarization linear combinations of powers of the form $\varphi^{\otimes k}$ are dense in this restriction (see for example Remark 2.5 in [9]).

\section{Main result}

\subsection{Heuristics: macroscopic dynamics}

The goal of this section is to provide some intuitions on the type of limiting field that we should expect for fields of order greater than one. We will start by considering the 
cases $k=1,2$ and, inspired by the results obtained in [11], we will propose a heuristic interpretation of the two SPDEs obtained as scaling limits and their relation. Based on this interpretation we will conjecture a possible generalization to the $k$ th-order case. In Section 5.2 we will give the rigorous result confirming the validity of the conjecture.

Here we will informally use the notation $\mathscr{Y}_{t}^{(k)}$ and $\mathscr{X}_{t}^{(k)}$ for the distributional limits of $\mathscr{Y}^{(n, k)}$ and $\mathscr{X}^{(n, k)}$ respectively.

Recall that from (3.13) we know that formally the distribution valued first order field $\mathscr{X}_{t}^{(1)}(x)$ is a solution to the Ornstein-Uhlenbeck equation

$$
d \mathscr{X}_{t}^{(1)}(x)=\frac{\chi \alpha}{2} \Delta \mathscr{X}_{t}^{(1)}(x) d t+c_{\sigma} \sqrt{\chi \rho(\alpha+\sigma \rho)} \nabla d \mathscr{W}_{t}(x)
$$

where for $x \in \mathbb{R}^{d}, \mathscr{W}_{t}(x)$ is a space-time white noise and $\nabla d \mathscr{W}_{t}(x)$ should be interpreted as in (3.8)-(3.9).

Additionally, from the martingale problem given in [11], we can deduce that the distribution-valued second-order field $\mathscr{X}_{t}^{(2)}(x, y)$ is a solution to the SPDE

$$
\begin{aligned}
d \mathscr{X}_{t}^{(2)}(x, y) & =\frac{\chi \alpha}{2} \Delta^{(2)} \mathscr{X}_{t}^{(2)}(x, y) d t+c_{\sigma} \sqrt{\chi \rho(\alpha+\sigma \rho)} \mathscr{X}_{t}^{(1)}(x) \nabla d \mathscr{W}_{t}(y) \\
& +c_{\sigma} \sqrt{\chi \rho(\alpha+\sigma \rho)} \mathscr{X}_{t}^{(1)}(y) \nabla d \mathscr{W}_{t}(x)
\end{aligned}
$$

where $\mathscr{W}_{t}(x)$ is the same white noise as in (5.1) and $\Delta^{(2)}$ is the usual $2 d$-dimensional Laplacian, which is the sum of the Laplacian in the $x$ variable plus the Laplacian in the $y$ variable.

The key idea to extrapolate these relations to higher orders is to interpret the nonlinearity on the RHS of (5.2) as some product of fields, that we denote by $\diamond$, that satisfies the Leibniz rule of differentiation. This interpretation suggests that the second-order field $\mathscr{X}_{t}^{(2)}(x, y)$ is, in turn, a second power of the first-order field $\mathscr{X}_{t}^{(1)}(x)$. More precisely conjecturing

$$
\mathscr{X}_{t}^{(2)}(x, y)=\mathscr{X}_{t}^{(1)}(x) \diamond \mathscr{X}_{t}^{(1)}(y),
$$

since the product $\diamond$ follows the Leibniz rule we would have that

$$
\begin{aligned}
d \mathscr{X}_{t}^{(2)}(x, y) & =d\left(\mathscr{X}_{t}^{(1)}(x) \diamond \mathscr{X}_{t}^{(1)}(y)\right) \\
& =d \mathscr{X}_{t}^{(1)}(x) \diamond \mathscr{X}_{t}^{(1)}(y)+\mathscr{X}_{t}^{(1)}(x) \diamond d \mathscr{X}_{t}^{(1)}(y) \\
& =\left(\frac{\chi \alpha}{2} \Delta \mathscr{X}_{t}^{(1)}(x) d t+c_{\sigma} \sqrt{\chi \rho(\alpha+\sigma \rho)} \nabla d \mathscr{W}_{t}(x)\right) \diamond \mathscr{X}_{t}^{(1)}(y) \\
& +\mathscr{X}_{t}^{(1)}(x) \diamond\left(\frac{\chi \alpha}{2} \Delta \mathscr{X}_{t}^{(1)}(y) d t+c_{\sigma} \sqrt{\chi \rho(\alpha+\sigma \rho)} \nabla d \mathscr{W}_{t}(y)\right) \\
& =\frac{\chi \alpha}{2} \Delta^{(2)} \mathscr{X}_{t}^{(2)}(x, y) d t+c_{\sigma} \sqrt{\chi \rho(\alpha+\sigma \rho)} \mathscr{X}_{t}^{(1)}(x) \diamond \nabla d \mathscr{W}_{t}(y) \\
& +c_{\sigma} \sqrt{\chi \rho(\alpha+\sigma \rho)} \mathscr{X}_{t}^{(1)}(y) \diamond \nabla d \mathscr{W}_{t}(x)
\end{aligned}
$$

which indeed agress with (5.2).

Remark 5.1. This section is created with the intention to develop some intuition on the type of martingale problem we should expect for higher-order fields. The precise product to be used in this section is not relevant since after all our derivations are just made at a formal level. What is important is that the product should satisfy the Leibniz rule.

After the discussion above, it seems natural to expect that the $k$ th-order field is a $k$ th $\diamond$-power of the first-order one. More precisely we conjecture that a relation of the type

$$
\mathscr{X}_{t}^{(k)}\left(x_{1}, x_{2}, \ldots, x_{k}\right)=\mathscr{X}_{t}^{(1)}\left(x_{1}\right) \diamond \mathscr{X}_{t}^{(1)}\left(x_{2}\right) \diamond \cdots \diamond \mathscr{X}_{t}^{(1)}\left(x_{k}\right)
$$


is satisfied. If this holds true, computations analogous to (5.3) would imply the formal SPDE

$$
d \mathscr{X}_{t}^{(k)}(\mathbf{x})=\frac{\chi \alpha}{2} \Delta^{(k)} \mathscr{X}_{t}^{(k)}(\mathbf{x}) d t+c_{\sigma} \sqrt{\chi \rho(\alpha+\sigma \rho)} \sum_{j=1}^{k} \mathscr{X}_{t}^{(k-1)}\left(\mathbf{x}^{-j}\right) \diamond \nabla d \mathscr{W}_{t}\left(x_{j}\right)
$$

where $\Delta^{(k)}$ is the $k d$-dimensional Laplacian, defined as the sum of the Laplacians at each coordinate and $\mathbf{x}^{-j}$ is the $(k-1) d$-dimensional vector obtained from $\mathbf{x}$ by removing its coordinate $x_{j}$.

In the following section we formulate rigorously the meaning of the heuristic equation, via a martingale problem.

\subsection{Main theorem}

Let us spend one paragraph to introduce the probability notions which are relevant for our main result. As we already mentioned, the $k$ th-order fluctuation field can be considered as taking values in $S^{\prime}\left(\mathbb{R}^{k d}\right)$, the space of tempered distributions which is dual to $S\left(\mathbb{R}^{k d}\right)$. Our original process $\eta_{n^{2} t}$ has state space $\Omega^{(n)}$ corresponding to the rescaled lattice $\frac{1}{n} \mathbb{Z}$. We then denote by $\mathbb{P}_{n}$, respectively $\mathbb{E}_{n}$, the probability measure, respectively expectation, induced by the measure $\nu_{\rho}$ and the diffusively rescaled process $\eta_{n^{2} t}$ on $D\left([0, T] ; \Omega^{(n)}\right)$. We also denote by $Q_{n}^{(k)}$ the probability measure on $D\left([0, T] ; S^{\prime}\left(\mathbb{R}^{k d}\right)\right)$ induced by the density fluctuation field $\mathscr{X}_{t}^{(n, k)}$ over $\mathbb{P}_{n}$.

In what follows we will view the trajectory of the limiting fields up to order $\mathrm{k}$ as a vector

$$
\mathbb{X}_{t}^{(k)}=\left(\mathscr{X}^{(1)}, \ldots, \mathscr{X}^{(k)}\right)(t)
$$

where $0 \leq T$. This means that the trajectory $\left\{\mathbb{X}_{t}^{(k)}: 0 \leq t \leq T\right\}$ can be seen as an element of the path-space:

$$
\bigotimes_{l=1}^{k} D\left([0, T] ; S^{\prime}\left(\mathbb{R}^{l d}\right)\right)
$$

where, for each $1 \leq l \leq k$, we have that $\mathscr{X}^{(l)}$ is an element of the path-space $D([0, T]$; $\left.S^{\prime}\left(\mathbb{R}^{l d}\right)\right)$.

Motivated by this point of view, we equip the product path-space

$$
\bigotimes_{l=1}^{k} D\left([0, T] ; S^{\prime}\left(\mathbb{R}^{l d}\right)\right)
$$

with the filtration $\left\{\mathscr{F}_{t}^{k}: t \geq 0\right\}$ generated by the projections

$$
\pi_{t, l, \varphi_{l}}\left(\omega_{1}, \omega_{2}, \ldots, \omega_{k}\right)=\omega_{l}(t)\left[\varphi_{l}\right]
$$

where for $1 \leq l \leq k$, we have that $\varphi_{l} \in S\left(\mathbb{R}^{l d}\right)$ is a symmetric Schwartz function.

Theorem 5.2. The process $\left\{\mathscr{X}_{t}^{(n, k)}: t \in[0, T]\right\}$ converges in distribution, with respect to the J1-topology of $D\left([0, T] ; S^{\prime}\left(\mathbb{R}^{k d}\right)\right)$, as $n \rightarrow \infty$ to the process $\left\{\mathscr{X}_{t}^{(k)}: t \in[0, T]\right\}$ being the unique solution of the following recursive martingale problem.

Recursive martingale problem: for any symmetric $\varphi^{(k)} \in S\left(\mathbb{R}^{k d}\right)$ the process

$$
M_{t}^{(k)}\left(\varphi^{(k)}\right)=\mathscr{X}_{t}^{(k)}\left(\varphi^{(k)}\right)-\mathscr{X}_{0}^{(k)}\left(\varphi^{(k)}\right)-\frac{\chi \alpha}{2} \int_{0}^{t} \mathscr{X}_{s}^{(k)}\left(\Delta^{(k)} \varphi^{(k)}\right) d s
$$


is a continuous $\mathscr{F}_{t}^{k}$-square integrable martingale of quadratic variation

$$
c_{\sigma}^{2} \chi \rho(\alpha+\sigma \rho) \int_{0}^{t} \int_{\mathbb{R}^{d}}\|\nabla \varphi(x)\|^{2}\left(\mathscr{X}_{s}^{(k-1)}\left(\varphi^{(k-1)}\right)\right)^{2} d x d s
$$

with initial condition $\mathscr{X}_{t}^{(1)}$ given by the solution of (5.1).

Remark 5.3. This recursive martingale problem is the rigorous counterpart of the formal SPDE (5.4) that we heuristically obtained. Moreover, the specification of the filtration $\mathscr{F}_{t}^{k}$ guarantees that the driving noise is the same.

\subsection{Strategy of the proof}

We will show Theorem 5.2 by using induction on $k$. In the proof we will take advantage of the fact that the base case, $k=1$, is already proved in the literature. On the other hand, the inductive step will be proven by means of an approach based on the natural Dynkin martingales:

$$
M_{t}^{(n, k)}(\Phi)=\mathscr{Y}_{t}^{(n, k)}(\Phi)-\mathscr{Y}_{0}^{(n, k)}(\Phi)-n^{2} \int_{0}^{t} \mathscr{L}_{s}^{(n, k)}(\Phi) d s
$$

and

$$
N_{t}^{(n, k)}(\Phi)=\left(M_{t}^{(n, k)}(\Phi)\right)^{2}-n^{2} \int_{0}^{t} \Gamma \mathscr{Y}_{s}^{(n, k)}(\Phi) d s
$$

where $\Gamma$ is the so-called carré-du-champ operator given by:

$$
\Gamma(f)=\mathscr{L}\left(f^{2}\right)-2 f \mathscr{L}(f) .
$$

Notice that the Dynkin martingales can also be expressed in terms of the fields $\mathscr{X}_{t}^{(n, k)}$.

Roughly our approach consists of the following steps:

1. we express the integrand term of equation (5.7) in terms of the $k$ th-order fluctuation field $\mathscr{Y}^{(n, k)}$ using duality (Section 6.1);

2. we close the equation (5.8) by expressing the integrand in the RHS in terms of the $(k-1)$ th-order fluctuation field $\mathscr{Y}^{(n, k-1)}$ (Section 6.2);

3. we show tightness for the sequence of probability measures $Q_{n}^{(k)}$ (Section 6.3);

4. finally we characterize the limiting field by showing uniqueness of the solution of the martingale problem (Sections 6.4-6.5).

\subsection{Inductive argument}

The proof is done by induction over the order of the field $k$. The base case $k=1$, corresponding to the density fluctuation field (3.1), is assumed to be true. Indeed, as mentioned in Section 3, a proof of Theorem 5.2 for exclusion dynamics and zero-range processes (of which independent random walkers are a particular case) is given in [6] and [12] respectively. By similar arguments the result can be extended to the case of inclusion process.

To implement the inductive argument we formalize the following inductive hypothesis that will be referred to several times in the course of the proof of Theorem 5.2.

INDUCTIVE HYPOTHESIS 5.1. For any $k_{0} \in\{1,2, \ldots, k-1\}$ the sequence $\left\{\mathscr{X}_{t}^{\left(n, k_{0}\right)}: t \in[0, T]\right\}$ converges in distribution, with respect to the J1-topology of $D\left([0, T] ; S^{\prime}\left(\mathbb{R}^{k_{0} d}\right)\right.$ ), as $n \rightarrow \infty$ to the process $\left\{\mathscr{X}_{t}^{\left(k_{0}\right)}: t \in[0, T]\right\}$ being the unique solution of the following martingale problem. 
Higher order fluctuation fields

Martingale problem: for any symmetric $\varphi^{\left(k_{0}\right)} \in S\left(\mathbb{R}^{k_{0} d}\right)$ the process

$$
M_{t}^{\left(k_{0}\right)}\left(\varphi^{\left(k_{0}\right)}\right)=\mathscr{X}_{t}^{\left(k_{0}\right)}\left(\varphi^{\left(k_{0}\right)}\right)-\mathscr{X}_{0}^{\left(k_{0}\right)}\left(\varphi^{\left(k_{0}\right)}\right)-\frac{\chi \alpha}{2} \int_{0}^{t} \mathscr{X}_{s}^{\left(k_{0}\right)}\left(\Delta^{\left(k_{0}\right)} \varphi^{\left(k_{0}\right)}\right) d s
$$

is a continuous square integrable martingale of quadratic variation

$$
c_{\sigma}^{2} \chi \rho(\alpha+\sigma \rho) \int_{0}^{t} \int_{\mathbb{R}^{d}}\|\nabla \varphi(x)\|^{2}\left(\mathscr{X}_{s}^{\left(k_{0}-1\right)}\left(\varphi^{\left(k_{0}-1\right)}\right)\right)^{2} d x d s .
$$

\section{Proof of Theorem 5.2}

\subsection{Closing the equation for the drift term: $k \geq 2$}

In order to close the equation (5.7) for the drift term (i.e., the integral term), thanks to Remark 4.2 we can just proceed as follows

$$
\begin{aligned}
n^{2} \mathscr{L} \mathscr{Y}^{(n, k)}(\Phi, \eta) & =n^{-k d / 2} \sum_{\xi \in \Omega_{k}} n^{2} \Phi_{n}(\xi) \cdot[\mathscr{L} \mathfrak{D}(\xi, \cdot)](\eta) \\
& =n^{-k d / 2} \sum_{\xi \in \Omega_{k}} n^{2} \Phi_{n}(\xi) \cdot \Lambda(\xi) \cdot[\mathscr{L} D(\xi, \cdot)](\eta) \\
& =n^{-k d / 2} \sum_{\xi \in \Omega_{k}} n^{2} \Phi_{n}(\xi) \cdot \Lambda(\xi) \cdot\left[\mathscr{L}^{(k)} D(\cdot, \eta)\right](\xi) \\
& =n^{-k d / 2} \sum_{\xi \in \Omega_{k}} n^{2}\left[\mathscr{L}^{(k)} \Phi_{n}\right](\xi) \cdot \Lambda(\xi) \cdot D(\xi, \eta) \\
& =n^{-k d / 2} \sum_{\xi \in \Omega_{k}} n^{2}\left[\mathscr{L}^{(k)} \Phi_{n}\right](\xi) \cdot \mathfrak{D}(\xi, \eta) .
\end{aligned}
$$

We proceed evaluating the action of the $k$-particles generator on $\Phi_{n}$. We then have

$$
\begin{aligned}
n^{2}\left[\mathscr{L}^{(k)} \Phi_{n}\right](\xi) & =\sum_{x \in \mathbb{Z}^{d}} \sum_{r \in \mathscr{R}} p(r) \cdot \xi_{x}\left(\alpha+\sigma \xi_{x+r}\right) \cdot n^{2}\left(\Phi_{n}\left(\xi^{x, x+r}\right)-\Phi_{n}(\xi)\right) \\
& =\sum_{x \in \mathbb{Z}^{d}} \Phi_{n}\left(\xi-\delta_{x}\right) \sum_{r \in \mathscr{R}} p(r) \cdot \xi_{x}\left(\alpha+\sigma \xi_{x+r}\right) \cdot n^{2}\left(\varphi\left(\frac{x+r}{n}\right)-\varphi\left(\frac{x}{n}\right)\right) \\
& =\alpha \sum_{x \in \mathbb{Z}^{d}} \Phi_{n}\left(\xi-\delta_{x}\right) \cdot \xi_{x} \sum_{r \in \mathscr{R}} p(r) \cdot n^{2}\left(\varphi\left(\frac{x+r}{n}\right)-\varphi\left(\frac{x}{n}\right)\right) \\
& +\sigma \sum_{x \in \mathbb{Z}^{d}} \Phi_{n}\left(\xi-\delta_{x}\right) \sum_{r \in \mathscr{R}} p(r) \cdot \xi_{x} \xi_{x+r} \cdot n^{2}\left(\varphi\left(\frac{x+r}{n}\right)-\varphi\left(\frac{x}{n}\right)\right)
\end{aligned}
$$

Remark 6.1. Notice that the contribution coming from the second term in the RHS of (6.1) does not appear in the case $k=1$.

First of all we prove that

$$
n^{2} \sum_{r \in \mathscr{R}} p(r)\left(\varphi\left(\frac{x+r}{n}\right)-\varphi\left(\frac{x}{n}\right)\right)=\frac{\chi}{2} \cdot \Delta \varphi\left(\frac{x}{n}\right)+\frac{1}{n} \psi_{n}\left(\frac{x}{n}\right)
$$

for a suitable $\psi_{n} \in S(\mathbb{R})$ such that

$$
\sup _{n} \frac{1}{n^{d}} \sum_{x \in \mathbb{Z}^{d}} \psi_{n}\left(\frac{x}{n}\right)<\infty
$$

To prove this we use the Taylor expansion:

$$
\varphi\left(\frac{x+r}{n}\right)-\varphi\left(\frac{x}{n}\right)=\frac{1}{n} \sum_{j=1}^{d} r_{j} \cdot \frac{\partial \varphi}{\partial x_{j}}\left(\frac{x}{n}\right)+\frac{1}{2 n^{2}} \sum_{j, \ell=1}^{d} r_{j} r_{\ell} \frac{\partial^{2} \varphi}{\partial x_{j} \partial x_{\ell}}\left(\frac{x}{n}\right)+\ldots
$$




\section{Higher order fluctuation fields}

and then

$$
\begin{aligned}
& n^{2} \sum_{r \in \mathscr{R}} p(r)\left(\varphi\left(\frac{x+r}{n}\right)-\varphi\left(\frac{x}{n}\right)\right) \\
& =n \sum_{j=1}^{d}\left(\sum_{r \in \mathscr{R}} r_{j} p(r)\right) \cdot \frac{\partial \varphi}{\partial x_{j}}\left(\frac{x}{n}\right)+\frac{1}{2} \sum_{j, \ell=1}^{d}\left(\sum_{r \in \mathscr{R}} r_{j} r_{\ell} p(r)\right) \frac{\partial^{2} \varphi}{\partial x_{j} \partial x_{\ell}}\left(\frac{x}{n}\right)+\ldots
\end{aligned}
$$

for some $\psi_{n}$ satisfying (6.3). From the assumption (2.2), it follows that:

$$
\sum_{r_{j}=-R}^{R} r_{j} p(r)=0
$$

thus, from the fact that $\mathscr{R}=[-R, R]^{d} \cap \mathbb{Z}^{d}$ we have

$$
\sum_{r \in \mathscr{R}} r_{j} p(r)=0 \quad \text { and } \quad \sum_{r \in \mathscr{R}} r_{j} r_{\ell} p(r)=0 \quad \text { for } j \neq \ell
$$

as a consequence,

$$
\begin{aligned}
& n^{2} \sum_{r \in \mathscr{R}} p(r)\left(\varphi\left(\frac{x+r}{n}\right)-\varphi\left(\frac{x}{n}\right)\right)=\frac{1}{2} \sum_{\ell=1}^{d}\left(\sum_{r \in \mathscr{R}} r_{\ell}^{2} p(r)\right) \frac{\partial^{2} \varphi}{\partial x_{\ell}^{2}}\left(\frac{x}{n}\right)+\frac{1}{n} \psi_{n}\left(\frac{x}{n}\right) \\
& =\frac{\chi}{2} \cdot \sum_{\ell=1}^{d} \frac{\partial^{2} \varphi}{\partial x_{\ell}^{2}}\left(\frac{x}{n}\right)+\frac{1}{n} \psi_{n}\left(\frac{x}{n}\right)
\end{aligned}
$$

from which it follows (6.2).

Now we have

$$
n^{2}\left[\mathscr{L}^{(k)} \Phi_{n}\right](\xi)=\alpha \sum_{x \in \mathbb{Z}^{d}} \Phi_{n}\left(\xi-\delta_{x}\right) \cdot \xi_{x} \cdot\left(\frac{\chi}{2} \cdot \Delta \varphi\left(\frac{x}{n}\right)+\frac{1}{n} \psi_{n}\left(\frac{x}{n}\right)\right)+E_{n}(\varphi, \xi)
$$

with

$$
E_{n}(\varphi, \xi):=\sigma \sum_{x \in \mathbb{Z}^{d}} \Phi_{n}\left(\xi-\delta_{x}\right) \sum_{r \in \mathscr{R}} p(r) \cdot \xi_{x} \xi_{x+r} \cdot n^{2}\left(\varphi\left(\frac{x+r}{n}\right)-\varphi\left(\frac{x}{n}\right)\right)
$$

then we have

$$
\begin{aligned}
& \mathscr{L} \mathscr{Y}^{(n, k)}(\Phi, \eta)-\frac{1}{n^{k d / 2}} \sum_{\xi \in \Omega_{k}} E_{n}(\varphi, \xi) \cdot \mathfrak{D}(\xi, \eta) \\
& =\frac{\alpha}{n^{k d / 2}} \sum_{\xi \in \Omega_{k}} \mathfrak{D}(\xi, \eta) \sum_{x \in \mathbb{Z}^{d}} \Phi_{n}\left(\xi-\delta_{x}\right) \cdot \xi_{x} \cdot\left(\frac{\chi}{2} \cdot \Delta \varphi\left(\frac{x}{n}\right)+\frac{1}{n} \psi_{n}\left(\frac{x}{n}\right)\right) .
\end{aligned}
$$

It is now convenient to pass to the coordinate notation to treat sums of the type:

$$
\sum_{\xi \in \Omega_{k}} \mathfrak{D}(\xi, \eta) \sum_{x \in \mathbb{Z}^{d}} \Phi_{n}\left(\xi-\delta_{x}\right) \cdot \xi_{x} \cdot \psi\left(\frac{x}{n}\right)
$$

for some $\psi \in S\left(\mathbb{R}^{d}\right)$. First of all we notice that summing over $\xi \in \Omega_{k}$ is the same as 
summing over $\mathbf{x} \in \mathbb{Z}^{k d}$ :

$$
\begin{aligned}
& \sum_{\xi \in \Omega_{k}} \mathfrak{D}(\xi, \eta) \sum_{x \in \mathbb{Z}^{d}} \Phi_{n}\left(\xi-\delta_{x}\right) \cdot \xi_{x} \cdot \psi\left(\frac{x}{n}\right) \\
= & \sum_{\mathbf{x} \in \mathbb{Z}^{k d}} \frac{1}{N(\xi(\mathbf{x}))} \cdot \mathfrak{D}(\xi(\mathbf{x}), \eta) \sum_{i=1}^{k} \Phi_{n}\left(\xi(\mathbf{x})-\delta_{x_{i}}\right) \cdot \psi\left(\frac{x_{i}}{n}\right) \\
= & \sum_{\mathbf{x} \in \mathbb{Z}^{k d}} \frac{\Lambda(\xi(\mathbf{x}))}{N(\xi(\mathbf{x}))} \cdot D(\xi(\mathbf{x}), \eta) \sum_{i=1}^{k} \psi\left(\frac{x_{i}}{n}\right) \prod_{\substack{\ell=1 \\
\ell \neq i}}^{k} \varphi\left(\frac{x_{\ell}}{n}\right) \\
= & k \sum_{\mathbf{x} \in \mathbb{Z}^{k d}} \Pi(\mathbf{x}) \cdot D(\xi(\mathbf{x}), \eta) \prod_{\ell=1}^{k-1} \varphi\left(\frac{x_{\ell}}{n}\right) \cdot \psi\left(\frac{x_{k}}{n}\right) \\
= & k n^{k d / 2} \mathscr{X}^{(n, k)}\left(\varphi^{(k-1)} \otimes \psi, \eta\right)
\end{aligned}
$$

where the last identity follows using the expression of the field acting on more general (i.e., non-symmetric) test functions (4.14). Then, substituting in (6.8) we get

$$
\begin{aligned}
& \mathscr{L}^{(n, k)}(\Phi, \eta)-\frac{1}{n^{k d / 2}} \sum_{\xi \in \Omega_{k}} E_{n}(\varphi, \xi) \cdot \mathfrak{D}(\xi, \eta) \\
= & \alpha k \mathscr{X}^{(n, k)}\left(\varphi^{(k-1)} \otimes\left(\frac{\chi}{2} \Delta \varphi+\frac{1}{n} \psi_{n}\right), \eta\right)
\end{aligned}
$$

where we used the fact that $\varphi$ is uniformly bounded on $\mathbb{Z}$. From this we can see that it is possible to close the equation for the second order fluctuation field, modulo an error term that we define as follows

$$
\mathscr{E}^{(n, k)}(\varphi, \eta):=\mathscr{L}^{(n, k)}(\Phi, \eta)-\alpha k \cdot \frac{\chi}{2} \cdot \mathscr{X}^{(n, k)}\left(\varphi^{(k-1)} \otimes \Delta \varphi, \eta\right) .
$$

Then we have

$$
\mathscr{E}^{(n, k)}(\varphi, \eta)=\mathscr{E}_{1}^{(n, k)}(\varphi, \eta)+\mathscr{E}_{2}^{(n, k)}(\varphi, \eta)
$$

with

$$
\mathscr{E}_{1}^{(n, k)}(\varphi, \eta):=\frac{\alpha k}{n} \mathscr{X}^{(n, k)}\left(\varphi^{(k-1)} \otimes \psi_{n}, \eta\right)
$$

and

$$
\mathscr{E}_{2}^{(n, k)}(\varphi, \eta):=\frac{1}{n^{k d / 2}} \sum_{\xi \in \Omega_{k}} E_{n}(\varphi, \xi) \mathfrak{D}(\xi, \eta)
$$

that has to be estimated. Analogously to the previous computation we have

$$
\begin{aligned}
E_{n}(\varphi, \xi(\mathbf{x})) & =\sigma n^{2} \sum_{i=1}^{k}\left(\prod_{\substack{\ell=1 \\
\ell \neq i}}^{k} \varphi\left(\frac{x_{\ell}}{n}\right)\right) \cdot \sum_{r \in \mathscr{R}} p(r)\left(\sum_{j=1}^{k} \mathbf{1}_{x_{j}=x_{i}+r}\right)\left(\varphi\left(\frac{x_{i}+r}{n}\right)-\varphi\left(\frac{x_{i}}{n}\right)\right) \\
& =\sigma n^{2} \sum_{i=1}^{k}\left(\prod_{\substack{\ell=1 \\
\ell \neq i}}^{k} \varphi\left(\frac{x_{\ell}}{n}\right)\right) \cdot \sum_{j=1}^{k} p\left(x_{j}-x_{i}\right)\left(\varphi\left(\frac{x_{j}}{n}\right)-\varphi\left(\frac{x_{i}}{n}\right)\right) \\
& =\sigma n^{2} \sum_{i, j=1}^{k}\left(\prod_{\substack{\ell=1 \\
\ell \neq i, j}}^{k} \varphi\left(\frac{x_{\ell}}{n}\right)\right) \cdot p\left(x_{j}-x_{i}\right) \varphi\left(\frac{x_{j}}{n}\right)\left(\varphi\left(\frac{x_{j}}{n}\right)-\varphi\left(\frac{x_{i}}{n}\right)\right) \\
& =\sigma \sum_{\substack{\{i, j\} \\
1 \leq i, j \leq k}}\left(\prod_{\substack{\ell=1 \\
\ell \neq i, j}}^{k} \varphi\left(\frac{x_{\ell}}{n}\right)\right) \cdot p\left(x_{j}-x_{i}\right) n^{2}\left(\varphi\left(\frac{x_{j}}{n}\right)-\varphi\left(\frac{x_{i}}{n}\right)\right)^{2}
\end{aligned}
$$


where in the last step we used the symmetry of $p(\cdot)$. Then

$$
\begin{aligned}
& \mathscr{E}_{2}^{(n, k)}(\varphi, \eta)= \\
& =\frac{1}{n^{k d / 2}} \sum_{\mathbf{x} \in \mathbb{Z}^{k d}} \Pi(\mathbf{x}) \cdot D(\xi(\mathbf{x}), \eta) \cdot E_{n}(\varphi, \xi(\mathbf{x})) \\
& =\frac{\sigma}{n^{k d / 2}} \sum_{\mathbf{x} \in \mathbb{Z}^{k d}} \Pi(\mathbf{x}) \cdot D(\xi(\mathbf{x}), \eta) \cdot \sum_{\substack{\{i, j\} \\
1 \leq i, j \leq k}}\left(\prod_{\substack{\ell=1 \\
\ell \neq i, j}}^{k} \varphi\left(\frac{x_{\ell}}{n}\right)\right) \cdot p\left(x_{j}-x_{i}\right) \cdot n^{2}\left(\varphi\left(\frac{x_{j}}{n}\right)-\varphi\left(\frac{x_{i}}{n}\right)\right)^{2} \\
& =\frac{k(k-1) \sigma}{2 n^{k d / 2}} \sum_{\mathbf{x} \in \mathbb{Z}^{k d}} \Pi(\mathbf{x}) \cdot D(\xi(\mathbf{x}), \eta) \cdot\left(\prod_{\ell=1}^{k-2} \varphi\left(\frac{x_{\ell}}{n}\right)\right) \cdot p\left(x_{k}-x_{k-1}\right) \cdot n^{2}\left(\varphi\left(\frac{x_{k-1}}{n}\right)-\varphi\left(\frac{x_{k}}{n}\right)\right)^{2} .
\end{aligned}
$$

Hence we have

$$
\mathscr{E}^{(n, k)}(\varphi, \eta)=\frac{k}{n^{k d / 2}} \sum_{\mathbf{x} \in \mathbb{Z}^{k d}} \Pi(\mathbf{x}) \cdot D(\xi(\mathbf{x}), \eta) \cdot \Psi_{n}(\mathbf{x})
$$

with

$$
\begin{aligned}
\Psi_{n}(\mathbf{x}) & :=\varphi^{(k-2)}\left(x_{1}, \ldots, x_{k-2}\right) \otimes\left(\frac{\alpha}{n} \varphi\left(x_{k-1}\right) \cdot \psi_{n}\left(\frac{x_{k}}{n}\right)\right. \\
& \left.+\frac{\sigma(k-1)}{2} p\left(x_{k}-x_{k-1}\right) n^{2}\left(\varphi\left(\frac{x_{k-1}}{n}\right)-\varphi\left(\frac{x_{k}}{n}\right)\right)^{2}\right) .
\end{aligned}
$$

It remains to show that the $L^{2}\left(\mathbb{P}_{n}\right)$ norm of $\mathscr{E}(n, k)\left(\varphi, \eta\left(n^{2} t\right)\right)$ vanishes in the limit as $n$ goes to infinity. This is done in the following lemma:

Lemma 6.2. Let $\mathscr{E}^{(n, k)}(\varphi, \eta)$ be given by (6.8), then, for every test function $\varphi \in \hat{S}\left(\mathbb{R}^{d}\right)$, the space of permutation-invariant Schwartz functions, there exists $C>0$ such that, for all $t \geq 0$ and $n \in \mathbb{N}$,

$$
\mathbb{E}_{n}\left[\left(\int_{0}^{t} \mathscr{E}^{(n, k)}\left(\varphi, \eta\left(n^{2} s\right)\right) d s\right)^{2}\right] \leq C \cdot \frac{t^{2}}{n}
$$

Proof. Using the fact that $\varphi$ is bounded and that $p(\cdot)$ has finite range we can conclude that there exists an $M>0$ such that

$$
\sup _{n} \sup _{\mathbf{x} \in \mathbb{Z}^{k d}}\left|\Psi_{n}(\mathbf{x})\right| \leq M \text {. }
$$

We recall here that the duality function is parametrized by the density parameter $\rho$, i.e. $D(\cdot, \cdot)=D_{\rho}(\cdot, \cdot)$ and that $\left\{D_{\rho}(\xi, \cdot), \xi \in \Omega\right\}$ is a family of products of polynomials that are orthogonal with respect to the reversible measure $\nu_{\rho}$. From the stationarity of $\nu_{\rho}$ we have

$$
\begin{array}{r}
\mathbb{E}_{n}\left[\left(\int_{0}^{t} \mathscr{E}^{(n, k)}\left(\varphi, \eta\left(n^{2} s\right)\right) d s\right)^{2}\right]=\int_{0}^{t} \int_{0}^{t} \mathbb{E}_{n}\left[\mathscr{E}^{(n, k)}\left(\varphi, \eta_{n^{2} s}\right) \mathscr{E}^{(n, k)}\left(\varphi, \eta_{n^{2} u}\right)\right] d u d s \\
=2 \int_{0}^{t} \int_{0}^{s} \int \mathbb{E}_{\eta}\left[\mathscr{E}^{(n, k)}\left(\varphi, \eta_{n^{2}(s-u)}\right)\right] \mathscr{E}^{(n, k)}(\varphi, \eta) \nu_{\rho}(d \eta) d u d s
\end{array}
$$

The fact that we can exchange expectations and integral is a consequence of Proposition 6.4 in Section 6.2.2, which does not use any results of the current section. 
Let us denote by $V_{n}(\varphi)$ the integrand in (6.15), then, using (2.15), we have

$$
\begin{aligned}
V_{n}(\varphi) & =\frac{1}{n^{k d}} \sum_{\mathbf{x}, \mathbf{y} \in \mathbb{Z}^{k d}} \Psi_{n}(\mathbf{x}) \Psi_{n}(\mathbf{y}) \cdot \Pi(\mathbf{x}) \Pi(\mathbf{y}) \cdot \int \mathbb{E}_{\eta}\left[D_{\rho}\left(\xi(\mathbf{x}), \eta_{n^{2}(s-u)}\right)\right] D_{\rho}(\xi(\mathbf{y}), \eta) \nu_{\rho}(d \eta) \\
& =\frac{1}{n^{k d}} \sum_{\mathbf{x}, \mathbf{y} \in \mathbb{Z}^{k d}} \Psi_{n}(\mathbf{x}) \Psi_{n}(\mathbf{y}) \cdot \Pi(\mathbf{x}) \Pi(\mathbf{y}) \cdot \frac{1}{\mu_{\rho}(\xi(\mathbf{y}))} \cdot p_{n^{2}(s-u)}(\xi(\mathbf{x}), \xi(\mathbf{y})) \\
& =\frac{c}{n^{k d}} \sum_{\mathbf{x} \in \mathbb{Z}^{k d}} \Psi_{n}(\mathbf{x}) \cdot \Pi(\mathbf{x}) \sum_{\mathbf{y} \in \mathbb{Z}^{k d}} \frac{1}{N(\xi(\mathbf{y}))} \cdot \Psi_{n}(\mathbf{y}) \cdot p_{n^{2}(s-u)}(\xi(\mathbf{x}), \xi(\mathbf{y})) \\
& \leq \frac{c M}{n^{k d}} \sum_{\mathbf{x} \in \mathbb{Z}^{k d}}\left|\Psi_{n}(\mathbf{x})\right| \cdot \Pi(\mathbf{x}) \sum_{\mathbf{y} \in \mathbb{Z}^{k d}} \frac{1}{N(\xi(\mathbf{y}))} \cdot p_{n^{2}(s-u)}(\xi(\mathbf{x}), \xi(\mathbf{y})) \\
& =\frac{c M}{n^{k d}} \sum_{\mathbf{x} \in \mathbb{Z}^{k d}}\left|\Psi_{n}(\mathbf{x})\right| \cdot \Pi(\mathbf{x}) \sum_{\xi^{\prime} \in \Omega_{k}} p_{n^{2}(s-u)}\left(\xi(\mathbf{x}), \xi^{\prime}\right) \\
& \leq \frac{c^{\prime} M}{n^{k d}} \sum_{\mathbf{x} \in \mathbb{Z}^{k d}}\left|\Psi_{n}(\mathbf{x})\right|
\end{aligned}
$$

where we used (2.15) in the second identity, (4.4) and (2.17) in the third identity (with $c=c(k, \rho))$ and (6.14) in the fourth line. From (6.12) we have

$$
\begin{aligned}
\frac{1}{n^{k d}} \sum_{\mathbf{x} \in \mathbb{Z}^{k d}}\left|\Psi_{n}(\mathbf{x})\right| & \leq \frac{\alpha}{n^{k d+1}} \sum_{\mathbf{x} \in \mathbb{Z}^{k d}}\left|\psi_{n}\right|\left(\frac{x_{k}}{n}\right) \cdot \prod_{\ell=1}^{k-1}\left|\varphi\left(\frac{x_{\ell}}{n}\right)\right| \\
& +\frac{\sigma(k-1)}{2 n^{k d}} \sum_{\mathbf{x} \in \mathbb{Z}^{k d}} \prod_{\ell=3}^{k}\left|\varphi\left(\frac{x_{\ell}}{n}\right)\right| \cdot p\left(x_{2}-x_{1}\right) n^{2}\left(\varphi\left(\frac{x_{2}}{n}\right)-\varphi\left(\frac{x_{1}}{n}\right)\right)^{2} .
\end{aligned}
$$

Using (6.3) we have that the first term in the r.h.s. of (6.17) is bounded by a constant times $n^{-1}$. For what concerns the second term, we have:

$$
\begin{aligned}
& \frac{\sigma(k-1)}{2 n^{(k-2) d}}\left(\prod_{\ell=3}^{k} \sum_{x_{\ell} \in \mathbb{Z}^{d}} \varphi\left(\frac{x_{\ell}}{n}\right)\right) \cdot \frac{1}{n^{2 d}} \sum_{x_{1}, x_{2} \in \mathbb{Z}^{d}} p\left(x_{2}-x_{1}\right) n^{2}\left(\varphi\left(\frac{x_{2}}{n}\right)-\varphi\left(\frac{x_{1}}{n}\right)\right)^{2} \\
& \leq \frac{c}{n^{2 d}} \sum_{x_{1}, x_{2} \in \mathbb{Z}^{d}} p\left(x_{2}-x_{1}\right) n^{2}\left(\varphi\left(\frac{x_{2}}{n}\right)-\varphi\left(\frac{x_{1}}{n}\right)\right)^{2} .
\end{aligned}
$$

Now, from the Taylor expansion (6.4) we know that there exists a sequence of functions where, using the fact that the range of $p(\cdot)$ is $\mathscr{R}=[-R, R]^{d}$, and the Taylor expansion (6.4) we have that there exists a smooth function $\widetilde{\psi} \in S\left(\mathbb{R}^{d}\right)$ such that, for all $x \in \mathbb{Z}^{d}$,

$$
\sup _{r \in \mathbb{R}}\left\{n^{2}\left(\varphi\left(\frac{x+r}{n}\right)-\varphi\left(\frac{x}{n}\right)\right)^{2}\right\} \leq \widetilde{\psi}\left(\frac{x}{n}\right)
$$

as a consequence we obtain the upper bound

$$
\begin{aligned}
& \frac{1}{n^{2 d}} \sum_{x_{1}, x_{2} \in \mathbb{Z}^{d}} p\left(x_{2}-x_{1}\right) n^{2}\left(\varphi\left(\frac{x_{2}}{n}\right)-\varphi\left(\frac{x_{1}}{n}\right)\right)^{2}=\frac{1}{n^{2 d}} \sum_{x \in \mathbb{Z}^{d}} \sum_{r \in \mathscr{R}} p(r) n^{2}\left(\varphi\left(\frac{x+r}{n}\right)-\varphi\left(\frac{x}{n}\right)\right)^{2} \\
& \leq \frac{1}{n^{2 d}} \sum_{r \in \mathscr{R}} \sum_{x \in \mathbb{Z}^{d}} p(r) \cdot \widetilde{\psi}\left(\frac{x}{n}\right) \leq \frac{c}{n^{2 d}} \sum_{x \in \mathbb{Z}^{d}} \widetilde{\psi}\left(\frac{x}{n}\right) \leq \frac{c^{\prime}}{n^{d}}
\end{aligned}
$$

where the inequality holds for a suitable $c^{\prime}>0$. In conclusion we have that there exists a constant $C>0$ such that

$$
V_{n}(\varphi) \leq \frac{C}{n}
$$

from which the statement follows. 
As a consequence of Lemma 6.2 we can close the drift term, i.e.

$$
\begin{aligned}
\mathscr{L}^{(n, k)}(\Phi, \eta) & =\alpha k \cdot \frac{\chi}{2} \cdot \mathscr{X}^{(n, k)}\left(\varphi^{(k-1)} \otimes \Delta \varphi, \eta\right)+\mathscr{E}^{(n, k)}(\varphi, \eta) \\
& =\alpha k \cdot \frac{\chi}{2} \cdot \mathscr{X}^{(n, k)}\left(\varphi^{(k-1)} \otimes \Delta \varphi, \eta\right)+O\left(n^{-1}\right) .
\end{aligned}
$$

\subsection{Closing the equation for the carré-du-champ}

In this section we will show that the integrand in the RHS of equation (5.8) can be expressed in terms of the $(k-1)$ th-order fluctuation field $\mathscr{Y}^{(n, k-1)}$. To achieve this we consider the expression for the carré-du-champ given by (7.2) in the Appendix. For the case of our $k$ th-order fluctuation field this becomes

$$
n^{2} \Gamma \mathscr{Y}^{(n, k)}(\Phi, \eta)=\frac{1}{n^{d}} \sum_{\substack{x \in \mathbb{Z}^{d} \\ r \in \mathscr{R}}} C(r, \eta)\left[n^{d / 2+1}\left(\mathscr{Y}^{(n, k)}\left(\Phi, \eta^{x, x+r}\right)-\mathscr{Y}^{(n, k)}(\Phi, \eta)\right)\right]^{2}
$$

where

$$
C(r, \eta)=p(r) \eta_{x}\left(\alpha+\sigma \eta_{x+r}\right) .
$$

Notice that here we multiplied by a factor $n^{d / 2+1}$ the squared term in order to cancel the $n^{2}$ in front of the carré-du-champ and get a general factor $n^{-d}$ in front of the sum.

In the next section we find some recursion relations for duality polynomials. The main application of these relations consists in allowing us to rewrite any polynomial depending on $\eta^{x, x+r}$ in terms of polynomials depending on the unmodified $\eta$.

\subsubsection{Recursion relation for duality polynomials}

In this section we obtain a recurrence relation for the single-site orthogonal polynomials. Before giving the result it is convenient to summarize the expression for the self-duality generating function by defining the function

$$
f_{\sigma}(t, n):=\sum_{m=0}^{\infty} \mathfrak{d}(m, n) \cdot t^{m}
$$

then $f_{\sigma}$ can be written in the form

$$
f_{\sigma}(t, n)=e_{\sigma}(t) \cdot h_{\sigma}(t)^{n}, \quad h_{\sigma}(t)=\frac{1+c_{\sigma} b_{\sigma} t}{1-\sigma^{2} t}, \quad e_{\sigma}(t)=\left\{\begin{array}{lll}
e^{-t} & \text { if } & \sigma=0 \\
(1-t)^{-\sigma \alpha} & \text { if } & \sigma= \pm 1
\end{array}\right.
$$

with $c_{\sigma}$ given by (3.11), and $b_{\sigma}$ is given as follows:

$$
b_{\sigma}=\left\{\begin{array}{lll}
1 & \text { if } & \sigma=0 \\
\alpha+\rho & \text { if } & \sigma=1 \\
\alpha-\rho & \text { if } & \sigma=-1
\end{array} .\right.
$$

Then we define the functions $g_{\sigma}, \tilde{g}_{\sigma}: \mathbb{N} \rightarrow \mathbb{R}$ given by

$$
\begin{aligned}
& g_{\sigma}(m):=\left.\frac{1}{m !} \frac{d^{m}}{d t^{m}} h_{\sigma}(t)\right|_{t=0} \quad \text { and } \quad \tilde{g}_{\sigma}(m):=\left.\frac{1}{m !} \frac{d^{m}}{d t^{m}} \frac{1}{h_{\sigma}(t)}\right|_{t=0} \quad \text { for } m \geq 1 \\
& \text { and } \quad g_{\sigma}(0)=\tilde{g}_{\sigma}(0):=1
\end{aligned}
$$


that are exactly computable:

$$
g_{\sigma}(m)=\left\{\begin{array}{ll}
\frac{1}{\rho} \cdot \mathbf{1}_{m=1} & \sigma=0 \\
-\frac{\alpha}{\rho} & \sigma=+1 \\
\frac{\alpha}{\rho} & \sigma=-1
\end{array} \quad \tilde{g}_{\sigma}(m)= \begin{cases}\left(\frac{1}{\rho}\right)^{m} & \sigma=0 \\
\left(-\frac{1}{\rho}\right)^{m-1} \cdot(\alpha+\rho)^{m-1}\left(-\frac{\alpha}{\rho}\right) & \sigma=+1 \\
\left(\frac{1}{\rho}\right)^{m-1} \cdot(\alpha-\rho)^{m-1}\left(\frac{\alpha}{\rho}\right) & \sigma=-1\end{cases}\right.
$$

for $m \geq 1$, that can be rewritten as

$$
\tilde{g}_{\sigma}(1)=c_{\sigma}=g_{\sigma}(1)
$$

and

$$
g_{\sigma}(m)=\left(c_{\sigma} b_{\sigma}+\sigma^{2}\right) \cdot \sigma^{2 m-2} \quad, \quad \tilde{g}_{\sigma}(m)=c_{\sigma}^{m-1} b_{\sigma}^{m-1}\left(c_{\sigma} b_{\sigma}+\sigma^{2}\right) \quad \text { for } m \geq 2.6
$$

We have the following result.

Theorem 6.3. For any $m, n \in \mathbb{N}$ we have

$$
\mathfrak{d}(m, n+1)=\sum_{j=0}^{m} g(m-j) \cdot \mathfrak{d}(j, n)
$$

and

$$
\mathfrak{d}(m, n-1)=\sum_{j=0}^{m} \tilde{g}(m-j) \cdot \mathfrak{d}(j, n)
$$

with $g, \tilde{g}: \mathbb{N} \rightarrow \mathbb{R}$ as in (6.26)-(6.28).

Proof. From (6.24) we have that

$$
f(t, n+1)=f(t, n) h(t)
$$

then, from the generating function definition (6.23), we deduce that

$$
\mathfrak{d}(m, n)=\left.\frac{1}{m !} \cdot \frac{d^{m}}{d t^{m}} f(t, n)\right|_{t=0}
$$

hence, the recurrence relation (6.31) and an application of Leibniz product rule for differentiation in the RHS above give

$$
\begin{aligned}
\mathfrak{d}(m, n+1) & =\left.\left.\frac{1}{m !} \cdot \sum_{j=0}^{m}\left(\begin{array}{c}
m \\
j
\end{array}\right) \frac{d^{j}}{d t^{j}} f(t, n)\right|_{t=0} \cdot \frac{d^{m-j}}{d t^{m-j}} h(t)\right|_{t=0} \\
& =\left.\frac{1}{m !} \cdot \sum_{j=0}^{m}\left(\begin{array}{c}
m \\
j
\end{array}\right) j ! \cdot \mathfrak{d}(j, n) \cdot \frac{d^{m-j}}{d t^{m-j}} h(t)\right|_{t=0} \\
& =\left.\sum_{j=0}^{m} \frac{1}{(m-j) !} \cdot \frac{d^{m-j}}{d t^{m-j}} h(t)\right|_{t=0} \cdot \mathfrak{d}(j, n) \\
& =\sum_{j=0}^{m} g(m-j) \cdot \mathfrak{d}(j, n)
\end{aligned}
$$

where in the second equality we used (6.32). This concludes the proof of (6.29). Equation (6.30) can be proved from the same reasoning, with the difference that we now have the inverse relation

$$
f(t, n-1)=f(t, n) \cdot \frac{1}{h(t)} .
$$


This change results, after the application of Leibniz rule, in the relation

$$
\begin{aligned}
\mathfrak{d}(m, n-1) & =\left.\frac{1}{m !} \cdot \sum_{j=0}^{m}\left(\begin{array}{c}
m \\
j
\end{array}\right) j ! \cdot \mathfrak{d}(j, n) \cdot \frac{d^{m-j}}{d t^{m-j}} \frac{1}{h(t)}\right|_{t=0} \\
& =\sum_{j=0}^{m} \tilde{g}(m-j) \cdot \mathfrak{d}(j, n)
\end{aligned}
$$

that concludes the proof.

\subsubsection{Controlling the moments of the fields}

The objective of this section is to take advantage of the ergodic properties of our process to introduce a result that will allow us to make multiple replacements, in the appropriate sense, inside the expression of the carré-du-champ given in (6.22). Let us start first with a uniform estimate for moments of the fields $\mathscr{Y}^{(n, l)}(\Phi, \eta)$.

Proposition 6.4. Let $l, m \in \mathbb{N}$ then we have

$$
\sup _{n \in \mathbb{N}} \mathbb{E}_{\nu_{\rho}}\left[\mathscr{Y}^{(n, l)}(\Phi, \eta)^{m}\right] \leq C(\rho, \varphi)
$$

Proof. As claimed in the statement of the proposition, this result holds for any finite natural number $m$. Nevertheless for simplicity we will only show how to obtain the estimates for $m \in\{2,4\}$ (which indeed are the only two uses that we make of this result). Let us start with the simplest non-trivial case, $m=2$, for which the result comes directly from orthogonality

$$
\begin{aligned}
\mathbb{E}_{\nu_{\rho}}\left[\mathscr{Y}^{(n, l)}(\Phi, \eta)^{2}\right] & =n^{-l d} \sum_{\xi, \xi^{\prime} \in \Omega_{l}} \Phi_{n}(\xi) \Phi_{n}\left(\xi^{\prime}\right) \Lambda(\xi) \Lambda\left(\xi^{\prime}\right) \mathbb{E}_{\nu_{\rho}}\left[D(\xi, \eta) D\left(\xi^{\prime}, \eta\right)\right] \\
& =n^{-l d} \sum_{\xi \in \Omega_{l}} \Phi_{n}(\xi)^{2} \Lambda(\xi)^{2} \frac{1}{\mu_{\rho}(\xi)} \\
& \leq K \cdot n^{-l d} \sum_{\xi \in \Omega_{l}} \Phi_{n}(\xi)^{2}<\infty
\end{aligned}
$$

where in the second line we used (2.13) and $K$ is given by

$$
K=\sup _{\xi \in \Omega_{k}} \frac{\Lambda(\xi)^{2}}{\mu_{\rho}(\xi)} .
$$

Notice that the previous estimate was possible due the fact that orthogonality, in the form of expression (2.13), allowed us to reduce the summation in the RHS of (6.35) from a $2 l d$ dimensional sum to an $l d$ dimensional sum in (6.36).

For the case $m=4$ we have

$$
\begin{aligned}
\mathbb{E}_{\nu_{\rho}}\left[\mathscr{Y}^{(n, l)}(\Phi, \eta)^{4}\right]= & n^{-2 l d} \sum_{\xi^{(j)} \in \Omega_{l}} \prod_{j=1}^{4} \Phi_{n}\left(\xi^{(j)}\right) \cdot \Lambda\left(\xi^{(j)}\right) \cdot \\
& \mathbb{E}_{\nu_{\rho}}\left[D\left(\xi^{(1)}, \eta\right) D\left(\xi^{(2)}, \eta\right) D\left(\xi^{(3)}, \eta\right) D\left(\xi^{(4)}, \eta\right)\right]
\end{aligned}
$$

For this case the sum in the RHS of (6.38) is $4 l d$-dimensional. Given the factor $n^{-2 l d}$ in front of the RHS, in order to obtain a uniform estimate, we would like this summation to be $2 l d$ dimensional instead. In order to see that this is indeed the case, we analyze the non-zero contribution coming from

$$
\mathbb{E}_{\nu_{\rho}}\left[D\left(\xi^{(1)}, \eta\right) D\left(\xi^{(2)}, \eta\right) D\left(\xi^{(3)}, \eta\right) D\left(\xi^{(4)}, \eta\right)\right] .
$$


By the product nature of the measure $\nu_{\rho}$ and the duality polynomials we have

$$
\begin{aligned}
\mathbb{E}_{\nu_{\rho}}\left[D\left(\xi^{(1)}, \eta\right) D\left(\xi^{(2)}, \eta\right) D\left(\xi^{(3)}, \eta\right) D\left(\xi^{(4)}, \eta\right)\right] \\
=\prod_{x \in \mathbb{Z}^{d}} \mathbb{E}_{\nu_{\rho}}\left[d\left(\xi_{x}^{(1)}, \eta\right) d\left(\xi_{x}^{(2)}, \eta\right) d\left(\xi_{x}^{(3)}, \eta\right) d\left(\xi_{x}^{(4)}, \eta\right)\right] .
\end{aligned}
$$

Notice that for every $x$ for which $\xi_{x}^{(j)}=0$ for all $j \in\{1,2,3,4\}$, the corresponding contribution in the RHS of (6.39) is equal to 1 and therefore negligible. This is precisely the reason why the summation in the RHS of (6.38) is at most $4 l d$-dimensional. We have indeed that the maximum number of $x \in \mathbb{Z}^{d}$ contributing to the product in the RHS of (6.39) is at most $4 l$, i.e. one for each of the $4 l$ particles that all the $\xi^{(j)}$ have in total. In reality we can see that there are less $x$ s giving a non-zero contribution. In order to see is, consider an $x \in \mathbb{Z}^{d}$ such that there exists a unique $j \in\{1,2,3,4\}$ for which $\xi_{x}^{(j)} \neq 0$. In this case, because of the zero mean of the single-site duality function we have

$$
\mathbb{E}_{\nu_{\rho}}\left[d\left(\xi_{x}^{(1)}, \eta\right) d\left(\xi_{x}^{(2)}, \eta\right) d\left(\xi_{x}^{(3)}, \eta\right) d\left(\xi_{x}^{(4)}, \eta\right)\right]=0
$$

this means that whenever $x \in \mathbb{Z}^{d}$ is such that there exists a $j \in\{1,2,3,4\}$ for which $\xi_{x}^{(j)} \neq 0$ there must be another $j^{\prime} \in\{1,2,3,4\}$ for which $\xi_{x}^{\left(j^{\prime}\right)} \neq 0$. In other words we only have a possibility of $2 l$ particles to distribute freely, and hence the summation in the RHS of (6.38) is at most $2 l d$-dimensional.

Proposition 6.5. Let $f: \mathbb{R}^{d} \rightarrow \mathbb{R}$ be a test function, and $\left\{M_{n}: \Omega \times \mathbb{R} \rightarrow \mathbb{R}: n \in \mathbb{N}\right\}$ be a sequence of uniformly bounded cylindrical functions of the form

$$
M_{n}(\eta, x)=f(x / n) \prod_{j \in \mathbb{N}} d\left(b_{j}, \eta_{x}\right)
$$

where only a finite number of $b_{j}$ are different from zero. Let also $\left\{a_{n}: n \in \mathbb{N}\right\}$ be a sequence of real numbers converging to 0 , we then have

$$
\lim _{n \rightarrow \infty} \mathbb{E}_{n}\left[\left(\int_{0}^{t} \frac{a_{n}}{n^{d}} \sum_{\substack{x \in \mathbb{Z}^{d} \\ r \in \mathscr{R}}} C\left(r, \eta\left(n^{2} s\right)\right) M_{n}\left(\eta_{x}\left(n^{2} s\right)\right) \cdot \mathscr{Y}^{(n, l)}\left(\Phi, \eta\left(n^{2} s\right)\right)^{m} d s\right)^{2}\right]=0
$$

for all $l \in\{1,2, \ldots, k-1\}$, and $m \in \mathbb{N}$.

Proof. By Cauchy-Schwarz we have

$$
\begin{aligned}
& \mathbb{E}_{n}\left[\left(\int_{0}^{t} \frac{a_{n}}{n^{d}} \sum_{\substack{x \in \mathbb{Z}^{d} \\
r \in \mathscr{R}}} C\left(r, \eta\left(n^{2} s\right)\right) \cdot M_{n}\left(\eta_{x}\left(n^{2} s\right)\right) \cdot \mathscr{Y}^{(n, l)}\left(\Phi, \eta\left(n^{2} s\right)\right)^{m} d s\right)^{2}\right] \\
\leq & \frac{a_{n}^{2} t}{n^{2 d}} \int_{0}^{t} \mathbb{E}_{n}\left[\mathscr{Y}^{(n, l)}\left(\Phi, \eta\left(n^{2} s\right)\right)^{2 m} \cdot\left(\sum_{\substack{x \in \mathbb{Z}^{d} \\
r \in \mathscr{R}}} C\left(r, \eta\left(n^{2} s\right)\right) \cdot M_{n}\left(\eta_{x}\left(n^{2} s\right)\right)\right)^{2}\right] d s \\
= & \frac{a_{n}^{2} t^{2}}{n^{2 d}} \mathbb{E}_{n}\left[\mathscr{Y}^{(n, l)}(\Phi, \eta)^{2 m} \cdot\left(\sum_{x \in \mathbb{Z}^{d}} \sum_{r \in \mathscr{R}} p(r) \eta_{x}\left(\alpha+\sigma \eta_{x+r}\right) \cdot M_{n}\left(\eta_{x}\right) \cdot\right)^{2}\right]
\end{aligned}
$$




$$
\begin{aligned}
& =\frac{a_{n}^{2} t^{2}}{n^{2 d}} \sum_{x, y \in \mathbb{Z}^{d}} \sum_{r_{1}, r_{2} \in \mathscr{R}} p\left(r_{1}\right) \cdot p\left(r_{2}\right) \cdot \mathbb{E}_{n}\left[M_{n}\left(\eta_{x}\right) \cdot M_{n}\left(\eta_{y}\right) \cdot \mathscr{Y}^{(n, l)}(\Phi, \eta)^{2 m}\right] \\
& \leq \frac{a_{n}^{2} t^{2}}{n^{2 d}} \sum_{x, y \in \mathbb{Z}^{d}} \sum_{r_{1}, r_{2} \in \mathscr{R}} p\left(r_{1}\right) \cdot p\left(r_{2}\right) \cdot \sqrt{\mathbb{E}_{n}\left[M_{n}\left(\eta_{x}\right)^{2} \cdot M_{n}\left(\eta_{y}\right)^{2}\right]} \cdot \sqrt{\mathbb{E}_{n}\left[\cdot \mathscr{Y}(n, l)(\Phi, \eta)^{4 m}\right]} \\
& \leq K t^{2} a_{n}^{2}
\end{aligned}
$$

where in the last line we used Proposition 6.4, the boundedness of the single-site duality polynomials $d\left(b_{j}, \eta_{x}\right)$ and the smoothness of $f$ in the representation (6.41). The result then follows from the convergence $a_{n} \rightarrow 0$.

\subsubsection{The gradient of the fluctuation fields}

Our goal for this section is to rewrite the square inside the RHS of (6.22) in terms of lower order fluctuation fields. We will see that this can be expressed, in agreement with (5.6), only in terms of the field of order $k-1$. Let us then denote by $\nabla_{d}^{i, i+r}$ the $d$-dimensional gradient

$$
\nabla_{d}^{i, i+r} \mathscr{Y}^{(n, k)}(\Phi, \eta)=n^{d / 2+1}\left(\mathscr{Y}^{(n, k)}\left(\Phi, \eta^{i, i+r}\right)-\mathscr{Y}^{(n, k)}(\Phi, \eta)\right)
$$

Notice that, by linearity of the $k$-th order field, we have

$$
\nabla^{i, j} \mathscr{Y}^{(n, k)}(\Phi, \eta):=n^{-\frac{(k-1) d}{2}+1} \sum_{\xi \in \Omega_{k}} \Phi_{n}(\xi)\left[\mathfrak{D}\left(\xi, \eta^{i, j}\right)-\mathfrak{D}(\xi, \eta)\right]
$$

with $\mathfrak{D}(\cdot, \cdot)$ as in (3.18). We define now, for $i, j \in \mathbb{Z}^{d}, \ell \leq k$, the auxiliary field

$$
\mathscr{Z}_{i, j}^{(n, k, \ell)}(\Phi, \eta):=n^{-k d / 2} \sum_{\xi \in \Omega_{k}} \mathbf{1}_{\xi_{i}+\xi_{j}=\ell} \cdot \Phi_{n}(\xi) \mathfrak{D}(\xi, \eta)
$$

then we have the following formula for the gradient of the fluctuation field.

\section{Proposition 6.6.}

$\nabla^{i, j} \mathscr{Y}^{(n, k)}(\Phi, \eta)=\sum_{s=1}^{k} n^{-\frac{(s-1) d}{2}} \cdot \sum_{m=1}^{s} n\left(\varphi\left(\frac{j}{n}\right)^{m}-\varphi\left(\frac{i}{n}\right)^{m}\right) \cdot g(m) \cdot \mathscr{Z}_{i, j}^{(n, k-m, s-m)}\left(\varphi, \eta-\delta_{i}\right)$

Proof. Using the product nature of the polynomials $\mathfrak{D}(\cdot, \eta)$ and of $\Phi_{n}(\cdot)$ we get

$$
\nabla^{i, j} \mathscr{Y}^{(n, k)}(\Phi, \eta)=n \sum_{s=1}^{k} n^{-\frac{(s-1) d}{2}} \cdot \mathscr{Z}_{i, j}^{(n, k-s, 0)}(\varphi, \eta) \cdot \sum_{a=0}^{s} Y_{i, j}^{(n, a, s-a)}(\varphi, \eta)
$$

and

$$
\begin{aligned}
Y_{i, j}^{(n, a, b)}(\varphi, \eta):=\varphi\left(\frac{i}{n}\right)^{a} \varphi\left(\frac{j}{n}\right)^{b}\left\{\mathfrak{d}\left(a, \eta_{i}-1\right) \mathfrak{d}\left(b, \eta_{j}+1\right)-\mathfrak{d}\left(a, \eta_{i}\right) \mathfrak{d}\left(b, \eta_{j}\right)\right\} \\
=\varphi\left(\frac{i}{n}\right)^{a} \varphi\left(\frac{j}{n}\right)^{b}\left\{\mathfrak{d}\left(a, \eta_{i}-1\right)\left[\mathfrak{d}\left(b, \eta_{j}+1\right)-\mathfrak{d}\left(b, \eta_{j}\right)\right]\right. \\
\left.\quad+\mathfrak{d}\left(b, \eta_{j}\right)\left[\mathfrak{d}\left(a, \eta_{i}-1\right)-\mathfrak{d}\left(a, \eta_{i}\right)\right]\right\}
\end{aligned}
$$




\section{Higher order fluctuation fields}

hence, using (6.29) we get

$$
\begin{aligned}
\sum_{a=0}^{s} Y_{i, j}^{(n, a, s-a)}(\varphi, \eta) & =\sum_{a=0}^{s-1} \varphi\left(\frac{i}{n}\right)^{a} \varphi\left(\frac{j}{n}\right)^{s-a} \mathfrak{d}\left(a, \eta_{i}-1\right)\left[\mathfrak{d}\left(s-a, \eta_{j}+1\right)-\mathfrak{d}\left(s-a, \eta_{j}\right)\right] \\
& -\sum_{b=0}^{s-1} \varphi\left(\frac{i}{n}\right)^{s-b} \varphi\left(\frac{j}{n}\right)^{b} \mathfrak{d}\left(b, \eta_{j}\right)\left[\mathfrak{d}\left(s-b, \eta_{i}\right)-\mathfrak{d}\left(s-b, \eta_{i}-1\right)\right] \\
& =\sum_{a=0}^{s-1} \sum_{\kappa=0}^{s-a-1} \varphi\left(\frac{i}{n}\right)^{a} \varphi\left(\frac{j}{n}\right)^{s-a} \cdot g(s-a-\kappa) \cdot \mathfrak{d}\left(a, \eta_{i}-1\right) \mathfrak{d}\left(\kappa, \eta_{j}\right) \\
& -\sum_{b=0}^{s-1} \sum_{m=0}^{s-b-1} \varphi\left(\frac{i}{n}\right)^{s-b} \varphi\left(\frac{j}{n}\right)^{b} \cdot g(s-b-m) \cdot \mathfrak{d}\left(m, \eta_{i}-1\right) \mathfrak{d}\left(b, \eta_{j}\right)
\end{aligned}
$$

now, calling $b=\kappa$ and $m=a$ we get

$$
\begin{aligned}
& \sum_{a=0}^{s-1} \sum_{\kappa=0}^{s-a-1} \varphi\left(\frac{i}{n}\right)^{a} \varphi\left(\frac{j}{n}\right)^{s-a} \cdot g(s-a-\kappa) \cdot \mathfrak{d}\left(a, \eta_{i}-1\right) \mathfrak{d}\left(\kappa, \eta_{j}\right) \\
& -\sum_{\kappa=0}^{s-1} \sum_{a=0}^{s-\kappa-1} \varphi\left(\frac{i}{n}\right)^{s-\kappa} \varphi\left(\frac{j}{n}\right)^{\kappa} \cdot g(s-a-\kappa) \cdot \mathfrak{d}\left(a, \eta_{i}-1\right) \mathfrak{d}\left(\kappa, \eta_{j}\right) \\
& =\sum_{a=0}^{s-1} \sum_{\ell=a}^{s-1}\left(\varphi\left(\frac{i}{n}\right)^{a} \varphi\left(\frac{j}{n}\right)^{s-a}-\varphi\left(\frac{i}{n}\right)^{s+a-\ell} \varphi\left(\frac{j}{n}\right)^{\ell-a}\right) \cdot g(s-\ell) \cdot \mathfrak{d}\left(a, \eta_{i}-1\right) \mathfrak{d}\left(\ell-a, \eta_{j}\right) \\
& =\sum_{a=0}^{s-1} \sum_{\ell=a}^{s-1}\left(\varphi\left(\frac{j}{n}\right)^{s-\ell}-\varphi\left(\frac{i}{n}\right)^{s-\ell}\right) \varphi\left(\frac{i}{n}\right)^{a} \varphi\left(\frac{j}{n}\right)^{\ell-a} \cdot g(s-\ell) \cdot \mathfrak{d}\left(a, \eta_{i}-1\right) \mathfrak{d}\left(\ell-a, \eta_{j}\right) \\
& =\sum_{\ell=0}^{s-1}\left(\varphi\left(\frac{j}{n}\right)^{s-\ell}-\varphi\left(\frac{i}{n}\right)^{s-\ell}\right) \cdot g(s-\ell) \sum_{a=0}^{\ell} \varphi\left(\frac{i}{n}\right)^{a} \varphi\left(\frac{j}{n}\right)^{\ell-a} \cdot \mathfrak{d}\left(a, \eta_{i}-1\right) \mathfrak{d}\left(\ell-a, \eta_{j}\right)
\end{aligned}
$$

where the first identity follows from the change of variable $\ell=\kappa+a$. Then

$$
\begin{aligned}
& \mathscr{Z}_{i, j}^{(n, k-s)}(\Phi, \eta) \cdot \sum_{a=0}^{s} Y_{i, j}^{(n, a, s-a)}(\varphi, \eta) \\
& =\sum_{\ell=0}^{s-1}\left(\varphi\left(\frac{j}{n}\right)^{s-\ell}-\varphi\left(\frac{i}{n}\right)^{s-\ell}\right) \cdot g(s-\ell) \cdot \mathscr{Z}_{i, j}^{(n, k-s, 0)}(\Phi, \eta) \cdot \\
& \sum_{a=0}^{\ell} \varphi\left(\frac{i}{n}\right)^{a} \varphi\left(\frac{j}{n}\right)^{\ell-a} \cdot \mathfrak{d}\left(a, \eta_{i}-1\right) \mathfrak{d}\left(\ell-a, \eta_{j}\right) \\
& =\sum_{\ell=0}^{s-1}\left(\varphi\left(\frac{j}{n}\right)^{s-\ell}-\varphi\left(\frac{i}{n}\right)^{s-\ell}\right) \cdot g(s-\ell) \cdot \mathscr{Z}_{i, j}^{(n, k-(s-\ell), \ell)}\left(\Phi, \eta-\delta_{i}\right)
\end{aligned}
$$

then

$$
\begin{aligned}
& \nabla^{i, j} \mathscr{Y}^{(n, k)}(\Phi, \eta) \\
& \quad=n \sum_{s=1}^{k} n^{-\frac{(s-1) d}{2}} \cdot \sum_{\ell=0}^{s-1}\left(\varphi\left(\frac{j}{n}\right)^{s-\ell}-\varphi\left(\frac{i}{n}\right)^{s-\ell}\right) \cdot g(s-\ell) \cdot \mathscr{Z}_{i, j}^{(n, k-(s-\ell), \ell)}\left(\Phi, \eta-\delta_{i}\right) \\
& \quad=\sum_{s=1}^{k} n^{-\frac{(s-1) d}{2}} \cdot \sum_{m=1}^{s} n\left(\varphi\left(\frac{j}{n}\right)^{m}-\varphi\left(\frac{i}{n}\right)^{m}\right) \cdot g(m) \cdot \mathscr{Z}_{i, j}^{(n, k-m, s-m)}\left(\Phi, \eta-\delta_{i}\right) .
\end{aligned}
$$

This concludes the proof. 


\section{Higher order fluctuation fields}

The advantage that Proposition 6.6 gives us is that we now have an expression in terms of the auxiliary field (6.45):

$$
\begin{aligned}
\nabla^{i, j} \mathscr{Y}^{(n, k)}(\Phi, \eta) & =\sum_{s=1}^{k} n^{-\frac{(s-1) d}{2}} \cdot \sum_{m=1}^{s} n\left(\varphi\left(\frac{j}{n}\right)^{m}-\varphi\left(\frac{i}{n}\right)^{m}\right) \cdot g(m) \cdot \mathscr{Z}_{i, j}^{(n, k-m, s-m)}\left(\Phi, \eta-\delta_{i}\right) \\
& =n\left(\varphi\left(\frac{j}{n}\right)-\varphi\left(\frac{i}{n}\right)\right) \cdot g(1) \cdot \mathscr{Z}_{i, j}^{(n, k-1,0)}\left(\Phi, \eta-\delta_{i}\right) \\
& +\sum_{s=2}^{k} n^{-\frac{(s-1) d}{2}} \cdot \sum_{m=1}^{s} n\left(\varphi\left(\frac{j}{n}\right)^{m}-\varphi\left(\frac{i}{n}\right)^{m}\right) \cdot g(m) \cdot \mathscr{Z}_{i, j}^{(n, k-m, s-m)}\left(\Phi, \eta-\delta_{i}\right) .
\end{aligned}
$$

Recall that we claimed that we are able to close the carré-du-champ in an expression depending only on the field of order $k-1$. In order to achieve this it remains to:

1. replace the first term in the RHS of (6.48) by some expressions depending on the field of order $k-1$;

2. show that the second term in the RHS of (6.48) vanishes as $n \rightarrow \infty$.

We will achieve this in several steps, the first one being the proof of the following proposition.

Proposition 6.7. For all $k \in \mathbb{N}$ we have

$\lim _{n \rightarrow \infty} \mathbb{E}_{n}\left[\left(\int_{0}^{t} \frac{1}{n^{d}} \sum_{\substack{x \in \mathbb{Z}^{d} \\ r \in \mathscr{R}}} C\left(r, \eta\left(n^{2} s\right)\right)\left(\mathscr{Z}_{x, x+r}^{(n, k, 0)}\left(\Phi, \eta\left(n^{2} s\right)\right)-\mathscr{Y}^{(n, k)}\left(\Phi, \eta\left(n^{2} s\right)\right)\right)^{2} d s\right)^{2}\right]=0$.

Proof. Notice that for any fixed $x$ we have

$$
\mathscr{Y}^{(n, k)}\left(\Phi, \eta\left(n^{2} s\right)\right)=\sum_{l=0}^{k} \mathscr{Z}_{x, x+r}^{(n, k, l)}\left(\Phi, \eta\left(n^{2} s\right)\right)
$$

which implies

$$
\begin{aligned}
\left(\mathscr{Z}_{x, x+r}^{(n, k, 0)}\left(\Phi, \eta\left(n^{2} s\right)\right)-\mathscr{Y}^{(n, k)}\left(\Phi, \eta\left(n^{2} s\right)\right)\right)^{2} & =\left(\sum_{l=1}^{k} \mathscr{Z}_{x, x+r}^{(n, k, l)}\left(\Phi, \eta\left(n^{2} s\right)\right)\right)^{2} \\
& \leq k \sum_{l=1}^{k} \mathscr{Z}_{x, x+r}^{(n, k, l)}\left(\Phi, \eta\left(n^{2} s\right)\right)^{2} .
\end{aligned}
$$

Moreover, we can also estimate each $\mathscr{Z}_{x, x+r}^{(n, k, l)}\left(\Phi, \eta\left(n^{2} s\right)\right)$ in terms of the coordinates field $\mathscr{X}^{(n, k-l)}$ given by (4.13) as follows:

$$
\mathscr{Z}_{x, x+r}^{(n, k, l)}(\Phi, \eta)^{2} \leq n^{-l d / 2} M_{n}(\eta, l) \cdot \mathscr{X}^{(n, k-l)}\left(\varphi^{(k-l)}, \eta\right)^{2}
$$

where $M_{n}$ is made of terms of the form (6.41), i.e.

$$
M_{n}(\eta, l)=\sum_{\xi_{x}=0}^{l} \Phi\left(\xi_{x} \delta_{x}+\left(l-\xi_{x}\right) \delta_{x+r}\right) \cdot d\left(\xi_{x}, \eta_{x}\right) \cdot d\left(l-\xi_{x}, \eta_{x+r}\right) .
$$

Thanks to Proposition 6.5 we conclude the proof. 
For what concerns the second step, let us denote by $\widehat{G}_{i, j}^{(n, k)}(\Phi, \eta)$ the second term in the RHS of (6.48), i.e.

$\widehat{G}_{i, j}^{(n, k)}(\Phi, \eta):=\sum_{s=2}^{k} n^{-\frac{(s-1) d}{2}} \cdot \sum_{m=1}^{s} n\left(\varphi\left(\frac{j}{n}\right)^{m}-\varphi\left(\frac{i}{n}\right)^{m}\right) \cdot g(m) \cdot \mathscr{Z}_{i, j}^{(n, k-m, s-m)}\left(\Phi, \eta-\delta_{i}\right)$

we have the following result supporting our claim:

Proposition 6.8. Under the inductive hypothesis 5.1 we have

$$
\lim _{n \rightarrow \infty} \mathbb{E}_{n}\left[\int_{0}^{t}\left(\frac{1}{n^{d}} \sum_{\substack{x \in \mathbb{Z}^{d} \\ r \in \mathscr{R}}} C\left(r, \eta\left(n^{2} s\right)\right) \cdot \widehat{G}_{x, x+r}^{(n, k)}\left(\varphi, \eta\left(n^{2} s\right)\right)^{2} d s\right)^{2}\right]=0 .
$$

Proof. After expanding $\widehat{G}_{x, x+r}^{(n, k)}\left(\varphi, \eta\left(n^{2} s\right)\right)^{2}$, the statement follows from applying multiple times Propositions 6.7 and 6.5.

Proposition 6.9. Let

$$
G_{i, j}^{(n, k)}(\varphi, \eta):=\nabla^{i, j} \mathscr{Y}^{(n, k)}(\Phi, \eta)+c_{\sigma}\left\langle j-i, \nabla \varphi\left(\frac{i}{n}\right)\right\rangle \cdot \mathscr{Y}^{(n, k-1)}(\Phi, \eta)
$$

then, under the inductive hypothesis 5.1, we have

$$
\lim _{n \rightarrow \infty} \mathbb{E}_{n}\left[\int_{0}^{t}\left(\frac{1}{n^{d}} \sum_{\substack{x \in \mathbb{Z}^{d} \\ r \in \mathscr{R}}} C\left(r, \eta\left(n^{2} s\right)\right) \cdot G_{x, x+r}^{(n, k)}\left(\varphi, \eta\left(n^{2} s\right)\right)^{2} d s\right)^{2}\right]=0 .
$$

Proof. Due to the fact that

$$
\mathscr{Z}_{i, j}^{(n, k-1,0)}\left(\Phi, \eta-\delta_{i}\right)=\mathscr{Z}_{i, j}^{(n, k-1,0)}(\Phi, \eta)
$$

if we isolate the term $s=1$ in (6.46) we obtain

$$
\nabla^{i, j} \mathscr{Y}^{(n, k)}(\Phi, \eta)=-c_{\sigma} n\left(\varphi\left(\frac{j}{n}\right)-\varphi\left(\frac{i}{n}\right)\right) \cdot \mathscr{Z}_{i, j}^{(n, k-1,0)}(\Phi, \eta)+\widehat{G}_{i, j}^{(n, k)}(\Phi, \eta)
$$

then the statement follows from Proposition 6.7 and Proposition 6.8.

\subsubsection{Conclusion}

From (6.22) and (6.54) we have

$$
\begin{aligned}
n^{2} & \Gamma \mathscr{Y}^{(n, k)}(\Phi, \eta) \\
& =\frac{1}{n^{d}} \sum_{x \in \mathbb{Z}^{d}} \sum_{r \in \mathscr{R}} p(r) \eta_{x}\left(\alpha+\sigma \eta_{x+r}\right)\left(c_{\sigma}\left\langle r, \nabla \varphi\left(\frac{x}{n}\right)\right\rangle \cdot \mathscr{Y}^{(n, k-1)}(\Phi, \eta)-G_{x, x+r}^{(n, k)}(\varphi, \eta)\right)^{2} \\
& =\frac{c_{\sigma}^{2}}{n^{d}}\left(\mathscr{Y}^{(n, k-1)}(\Phi, \eta)\right)^{2} \cdot \sum_{x \in \mathbb{Z}^{d}} \sum_{r \in \mathscr{R}}\left|\left\langle r, \nabla \varphi\left(\frac{x}{n}\right)\right\rangle\right|^{2} p(r) \eta_{x}\left(\alpha+\sigma \eta_{x+r}\right)+\mathscr{G}_{1}^{(n, k)}(\Phi, \eta)
\end{aligned}
$$

with

$$
\begin{aligned}
\mathscr{G}_{1}^{(n, k)}(\Phi, \eta):=\frac{1}{n^{d}} \sum_{x \in \mathbb{Z}^{d}} \sum_{r \in \mathscr{R}} p(r) \eta_{x}\left(\alpha+\sigma \eta_{x+r}\right) \cdot G_{x, x+r}^{(n, k)}(\varphi, \eta) \\
\cdot\left(G_{x, x+r}^{(n, k)}(\varphi, \eta)-2 c_{\sigma}\left\langle r, \nabla \varphi\left(\frac{x}{n}\right)\right\rangle \mathscr{Y}^{(n, k-1)}(\Phi, \eta)\right)
\end{aligned}
$$


then we can write

$$
\begin{aligned}
& n^{2} \Gamma \mathscr{Y}^{(n, k)}(\Phi, \eta)= \\
& =\rho(\alpha+\sigma \rho) \frac{c_{\sigma}^{2}}{n^{d}}\left(\mathscr{Y}^{(n, k-1)}(\Phi, \eta)\right)^{2} \cdot \sum_{\substack{x \in \mathbb{Z}^{d} \\
r \in \mathscr{R}}}\left|\left\langle r, \nabla \varphi\left(\frac{x}{n}\right)\right\rangle\right|^{2} p(r)+\mathscr{G}_{1}^{(n, k)}(\Phi, \eta)+\mathscr{G}_{2}^{(n, k)}(\varphi, \eta)
\end{aligned}
$$

with

$$
\begin{aligned}
& \mathscr{G}_{2}^{(n, k)}(\varphi, \eta):= \\
& \frac{c_{\sigma}^{2}}{n^{d}}\left(\mathscr{Y}^{(n, k-1)}(\Phi, \eta)\right)^{2} \cdot \sum_{x \in \mathbb{Z}^{d}} \sum_{r \in \mathscr{R}}\left|\left\langle r, \nabla \varphi\left(\frac{x}{n}\right)\right\rangle\right|^{2} p(r)\left\{\alpha\left(\eta_{x}-\rho\right)+\sigma\left(\eta_{x} \eta_{x+r}-\rho^{2}\right)\right\} .
\end{aligned}
$$

We first estimate the term due to the error $\mathscr{G}_{1}^{(n, k)}(\Phi, \eta)$.

Proposition 6.10. For every $t>0$ and every test function $\varphi \in S(\mathbb{R})$ there exists $C>0$ such that, for all $n \in \mathbb{N}$,

$$
\lim _{n \rightarrow \infty} \mathbb{E}_{n}\left[\left(\int_{0}^{t} \mathscr{G}_{1}^{(n, k)}\left(\Phi, \eta\left(n^{2} s\right)\right) d s\right)^{2}\right]=0
$$

Proof. It follows from Proposition 6.8 and the convergence, by inductive hypothesis, of $\mathscr{Y}^{(n, k-1)}(\varphi, \eta)$.

The two following propositions allow us to estimate the error $\mathscr{G}_{2}^{(n, k)}$ and then to perform the replacement in (6.57).

Lemma 6.11. For every $t>0$ and every test function $\varphi \in S(\mathbb{R})$ there exists $C>0$ such that, for all $n \in \mathbb{N}$,

$$
\lim _{n \rightarrow \infty} \mathbb{E}_{n}\left[\left(\int_{0}^{t} \frac{1}{n^{d}} \sum_{x \in \mathbb{Z}^{d}} \sum_{r \in \mathscr{R}}\left|\left\langle r, \nabla \varphi\left(\frac{x}{n}\right)\right\rangle\right|^{2} p(r)\left(\eta_{x}\left(n^{2} s\right)-\rho\right) d s\right)^{2}\right]=0 .
$$

Proof. From (3.10) we can write the integrand in (6.60) as

$$
\frac{1}{n^{d / 2}} \mathscr{Y}_{s}^{(n, 1)}(\Psi), \quad \text { with } \quad \Psi(\xi):=\prod_{x \in \mathbb{Z}^{d}} \psi(x)^{\xi_{x}} \quad \psi(x):=\sum_{r \in \mathscr{R}}|\langle r, \nabla \varphi(x)\rangle|^{2} p(r)(6.61)
$$

then the statement follows from the convergence of $\mathscr{Y}_{s}^{(n, 1)}(\Psi)$ and the extra factor $\frac{1}{n^{d / 2}}$.

Similarly, another replacement is necessary on the second term of the RHS of (6.58).

Lemma 6.12. For every $t>0$ and every test function $\varphi$ we have

$$
\lim _{n \rightarrow \infty} \mathbb{E}_{n}\left[\left(\int_{0}^{t} \frac{1}{n^{d}} \sum_{x \in \mathbb{Z}^{d}} \sum_{r \in \mathscr{R}}\left|\left\langle r, \nabla \varphi\left(\frac{x}{n}\right)\right\rangle\right|^{2} p(r)\left(\eta_{x}\left(n^{2} s\right)-\rho\right)\left(\eta_{x+r}\left(n^{2} s\right)-\rho\right) d s\right)^{2}\right]=0 .
$$

Proof. The proof of this lemma is done in the same spirit than Proposition 6.5.

Proposition 6.13. For every $t>0$ and every test function $\varphi \in S(\mathbb{R})$ there exists $C>0$ such that, for all $n \in \mathbb{N}$,

$$
\lim _{n \rightarrow \infty} \mathbb{E}_{n}\left[\left(\int_{0}^{t} \mathscr{G}_{2}^{(n, k)}\left(\Phi, \eta\left(n^{2} s\right)\right) d s\right)^{2}\right]=0
$$


Proof. It follows from Lemma 6.11, Lemma 6.12 and the convergence, by inductive hypothesis, of $\mathscr{Y}^{(n, k-1)}(\varphi, \eta)$.

From Propositions 6.10 and 6.13 we can write

$$
\begin{aligned}
& n^{2} \Gamma \mathscr{Y}^{(n, k)}(\Phi, \eta)= \\
& =\rho(\alpha+\sigma \rho) \frac{c_{\sigma}^{2}}{n^{d}}\left(\mathscr{Y}^{(n, k-1)}(\Phi, \eta)\right)^{2} \cdot \sum_{x \in \mathbb{Z}^{d}} \sum_{r \in \mathscr{R}}\left|\left\langle r, \nabla \varphi\left(\frac{x}{n}\right)\right\rangle\right|^{2} p(r)+\mathscr{G}^{(n, k)}(\Phi, \eta)
\end{aligned}
$$

where the term $\mathscr{G}^{(n, k)}(\Phi, \eta)$ is a vanishing error:

$$
\lim _{n \rightarrow \infty} \mathbb{E}_{n}\left[\left(\int_{0}^{t} \mathscr{G}^{(n, k)}\left(\Phi, \eta\left(n^{2} s\right)\right) d s\right)^{2}\right]=0 .
$$

Therefore we conclude that the proposed (remember that at this point we do not know if the limiting object is indeed a martingale) predictable quadratic variation of our limiting martingale is given by

$$
c_{\sigma}^{2} \chi \rho(\alpha+\sigma \rho) t\left(\mathscr{X}_{s}^{(k-1)}\left(\varphi^{(k-1)}\right)\right)^{2} \int_{\mathbb{R}^{d}}\|\nabla \varphi(x)\|^{2} d x
$$

Arrived at this point we can conclude that if $\left\{M_{t}^{(n, k)}(\Phi): t \in[0, T]\right\}$ has a limit as $n \rightarrow \infty$, and if the limit is a square-integrable martingale then its quadratic variation is given by (6.65). In what follows we will show tightness and uniform integrability, i.e. we will prove that $\left\{M_{t}^{(n, k)}(\Phi): t \in[0, T]\right\}$ converges to $\left\{M_{t}^{(k)}(\Phi): t \in[0, T]\right\}$ and that $\left\{M_{t}^{(k)}(\Phi): t \in[0, T]\right\}$ is indeed a martingale.

\subsection{Tightness}

In this section we prove tightness for the family of laws $\left\{Q_{n}^{(k)}\right\}_{n \in \mathbb{N}}$, induced by $\left\{\mathscr{X}^{(n, k)}(\cdot, t)\right\}_{t \geq 0}$ on $D\left([0, \infty), S^{\prime}\left(\mathbb{R}^{k}\right)\right)$. From the Dynkin formula we know that

$$
M_{n}^{\prime}\left(t, \varphi^{(k)}\right)=\mathscr{X}_{t}^{(n, k)}\left(\varphi^{(k)}\right)-n^{2} \int_{0}^{t} \mathscr{L}_{s}^{(n, k)}\left(\varphi^{(k)}\right) d s
$$

and

$$
N_{n}^{\prime}\left(t, \varphi^{(k)}\right)=M_{n}^{\prime}\left(t, \varphi^{(k)}\right)^{2}-n^{2} \int_{0}^{t} \Gamma \mathscr{X}_{s}^{(n, k)}\left(\varphi^{(k)}\right) d s
$$

are martingales. Theorem 2.3 in [8], which we include in Appendix 7.2, allows us to reduce the proof the tightness of $\left\{Q_{n}^{(k)}\right\}_{n \in \mathbb{N}}$ to the verification of conditions (7.5)-(7.7). We verify these conditions in Proposition 6.14, Proposition 6.15 and Proposition 6.16 below.

\subsubsection{The $\gamma_{1}$ term}

The following Proposition shows that conditions (7.5) and (7.6) hold true.

Proposition 6.14. For any $\varphi^{(k)} \in S\left(\mathbb{R}^{k d}\right)$ and $t_{0} \geq 0$ we have:

$$
\sup _{n \in \mathbb{N}} \sup _{0 \leq t \leq t_{0}} \mathbb{E}_{n}\left[\left(\mathscr{X}_{t}^{(n, k)}\left(\varphi^{(k)}\right)\right)^{2}\right]<\infty
$$

and

$$
\sup _{n \in \mathbb{N}} \sup _{0 \leq t \leq t_{0}} \mathbb{E}_{n}\left[\left(n^{2} \mathscr{L} \mathscr{X}_{t}^{(n, k)}\left(\varphi^{(k)}\right)\right)^{2}\right]<\infty
$$


Proof. We start with the proof (6.69) which is more involved. Thanks to stationarity, the expectation does not depend on time, and then we can ignore the supremum over time in (6.69). From (6.21) we already have an expression for the integrand of (6.69):

$$
n^{2} \mathscr{L} \mathscr{X}^{(n, k)}\left(\varphi^{(k)}, \eta\right)=\alpha k \cdot \frac{\chi}{2} \cdot \mathscr{X}^{(n, k)}\left(\varphi^{(k-1)} \otimes \Delta \varphi, \eta\right)+O\left(n^{-1}\right)
$$

recall that here again we are using the fact that the field $\mathscr{X}^{(n, k)}$ can be also thought as acting on general (not necessarily symmetric) test functions. Because of stationarity it is enough to estimate

$$
\mathbb{E}_{\nu_{\rho}}\left[\left(\mathscr{X}^{(n, k)}\left(\varphi^{(k-1)} \otimes \Delta \varphi, \eta\right)\right)^{2}\right]
$$

then the desired bound is obtained by applying Proposition 6.4. In the same spirit we can use Proposition 6.4 to bound (6.68).

\subsubsection{The $\gamma_{2}$ term}

Similarly to the previous section, here we prove the following proposition in order to verify the condition (7.6) for $\gamma_{2}$.

Proposition 6.15. For any $\varphi^{(k)} \in S\left(\mathbb{R}^{k d}\right)$ and $t_{0} \geq 0$ we have:

$$
\sup _{n \in \mathbb{N}} \sup _{0 \leq t \leq t_{0}} \mathbb{E}_{n}\left[\left(n^{2} \Gamma \mathscr{X}_{t}^{(n, k)}\left(\varphi^{(k)}\right)\right)^{2}\right]<\infty .
$$

Proof. Thanks to stationarity we can neglect the supremum over time. Recall that in (6.64) we have obtained an expression for the integrand on (6.72)

$$
\begin{aligned}
n^{2} \Gamma \mathscr{X}^{(n, k)}\left(\varphi^{(k)}, \eta\right) & = \\
& \rho(\alpha+\sigma \rho) \frac{k^{2} c_{\sigma}^{2}}{n^{d}}\left(\mathscr{Y}^{(n, k-1)}(\Phi, \eta)\right)^{2} \cdot \sum_{x \in \mathbb{Z}^{d}} \sum_{r \in \mathscr{R}}\left|\left\langle r, \nabla \varphi\left(\frac{x}{n}\right)\right\rangle\right|^{2} p(r)+O\left(n^{-1}\right)
\end{aligned}
$$

taking the square of which we obtain

$$
\begin{aligned}
& \mathbb{E}_{\nu_{\rho}}\left[\left(n^{2} \Gamma \mathscr{X}^{(n, k)}\left(\varphi^{(k)}, \eta\right)\right)^{2}\right] \\
= & \rho^{2}(\alpha+\sigma \rho)^{2} \frac{k^{4} c_{\sigma}^{4}}{n^{2 d}} \cdot \sum_{x, y \in \mathbb{Z}^{d}} \sum_{r_{1}, r_{2} \in \mathscr{R}}\left|\left\langle r_{1}, \nabla \varphi\left(\frac{x}{n}\right)\right\rangle\right|^{2} \cdot\left|\left\langle r_{2}, \nabla \varphi\left(\frac{y}{n}\right)\right\rangle\right|^{2} p\left(r_{1}\right) \cdot p\left(r_{2}\right) \\
\times & \mathbb{E}_{\nu_{\rho}}\left[\left(\mathscr{Y}^{(n, k-1)}(\Phi, \eta)\right)^{4}\right] .
\end{aligned}
$$

Notice that the first factor on the RHS of (6.73) can be controlled by using the compact support of $\varphi$ and the factor $\frac{1}{n^{2 d}}$. It is then sufficient to estimate

$$
\sup _{n \in \mathbb{N}} \mathbb{E}_{\nu_{\rho}}\left[\left(\mathscr{Y}^{(n, k-1)}(\Phi, \eta)\right)^{4}\right]
$$

then Proposition 6.4 finishes the proof.

\subsubsection{Modulus of continuity}

In this section we show that condition (2.5) of Theorem 2.3 in [8] is satisfied.

Proposition 6.16. For every $\varphi^{(k)} \in S\left(\mathbb{R}^{k d}\right)$ there exists a sequence $\delta(t, \varphi, n)$ converging to zero as $n \rightarrow 0$ such that:

$$
\lim _{n \rightarrow \infty} \mathbb{P}_{n}\left(\sup _{0 \leq t \leq T}\left|\mathscr{X}_{t}^{(n, k)}\left(\varphi^{(k)}, \eta\right)-\mathscr{X}_{t-}^{(n, k)}\left(\varphi^{(k)}, \eta\right)\right| \geq \delta(t, \varphi, n)\right)=0 .
$$


Proof. We know that the jumps of the process $\{\eta(t): t \geq 0\}$ are determined by exponential clocks. This implies that for any $\epsilon>0$ the probability of having more than one jump in the interval $(t, t+\epsilon]$ is of the order $o(\epsilon)$. Hence for $C$, a positive constant that depends on the model parameters, we have

$$
\sup _{0 \leq t \leq T}\left|\mathscr{X}_{t}^{(n, k)}\left(\varphi^{(k)}\right)-\mathscr{X}_{t-}^{(n, k)}\left(\varphi^{(k)}\right)\right| \leq C \frac{\|\varphi\|_{\infty}}{n^{k d / 2}}
$$

with probability $1-o(\epsilon)$.

Taking the sequence $\{\delta(t, \varphi, n)\}_{n \geq 1}$ given by

$$
\delta(t, \varphi, n)=C \frac{\|\varphi\|_{\infty}+1}{n^{1 / 2}}
$$

finishes the proof.

Remark 6.17. Notice that Proposition 6.16 implies, in particular, that the law induced by $\mathscr{Y}_{t}^{(n, k)}$ is concentrated on continuous paths.

\subsection{Characterization of limit points}

At this point we can only say that the sequence $\left\{M_{t}^{(n, k)}(\cdot): t \in[0, T]\right\}$ converges weakly to the process $\left\{M_{t}^{(k)}(\cdot): t \in[0, T]\right\}$ satisfying expressions (5.5) and (5.6). Nevertheless, we would like to support the claim, given in Theorem 5.2, that the limiting process $\left\{M_{t}^{(k)}(\cdot): t \in[0, T]\right\}$ is indeed a martingale with the proposed predictable quadratic variation given by (6.65). At this aim we prove the following result.

Proposition 6.18. The sequence $\left\{M_{t}^{(n, k)}(\cdot): t \in[0, T]\right\}$ is uniformly integrable.

Proof. By standard arguments it is enough to provide a uniform $L^{p}\left(\mathbb{P}_{n}\right)$ bound for $p>1$. Notice that, thanks to the martingale decomposition (5.7), and the same type of arguments used in the proofs of Propositions 6.14 and 6.15, we can indeed find the desired bounds for $p=2$.

The same type of reasoning used in Proposition 6.18 gives us the following result.

Proposition 6.19. The sequence $\left\{N_{t}^{(n, k)}(\cdot): t \in[0, T]\right\}$ is uniformly integrable.

Combining Propositions 6.18 and 6.19 we show that any limit point of the sequence $\left\{M_{t}^{(n, k)}(\cdot): t \in[0, T]\right\}$ satisfies the recursive martingale problem (5.5)-(5.6).

\subsection{Uniqueness}

It remains to show uniqueness of the solution of the martingale problem (5.5)-(5.6). First notice that by the Duhamel formula, from (5.5), we can deduce

$$
\mathscr{X}_{t}^{(k)}\left(\varphi^{(k)}\right)=\mathscr{X}_{0}^{(k)}\left(S_{t}^{(k)} \varphi^{(k)}\right)+\int_{0}^{t} d M_{s}^{(k)}\left(S_{t-s}^{(k)} \varphi^{(k)}\right)
$$

where $S_{t}^{(k)}$ is the semigroup associated to the $k d$-dimensional Laplacian (or to the $k d$ dimensional Brownian motion).

Remark 6.20. Notice that there is not ambiguity in using (5.5)-(5.6) with test functions of the form $S_{t}^{(k)} \varphi^{(k)}$. From the fact that the $d$-dimensional Brownian semigroup leaves invariant the space $S\left(\mathbb{R}^{d}\right)$, we can deduce that the $k d$-dimensional Brownian semigroup keeps both the symmetry and the Schwartz space nature of the test function $\varphi^{(k)}$. More precisely:

$$
S_{t}^{(k)} \varphi^{(k)}=\left(S_{t}^{(1)} \varphi\right)^{(k)}
$$

where $S_{t}^{(1)}$ denotes the semigroup of a $d$-dimensional Brwonian motion. 
Remark 6.21. In the RHS of equation (6.78), the integral term

$$
\int_{0}^{t} d M_{s}^{(k)}\left(S_{t-s}^{(k)} \varphi^{(k)}\right)
$$

should be interpreted as a martingale with quadratic variation

$$
\int_{0}^{t} \int_{\mathbb{R}^{d}}\left\|\nabla S_{t-s}^{(1)} \varphi(x)\right\|^{2} \mathscr{X}_{s}^{(k-1)}\left(S_{t-s}^{(k-1)} \varphi^{(k-1)}\right) d s .
$$

Given the distribution of $\mathscr{X}_{0}^{(k)}$ and the well-definedness of $M_{t}^{(k)}$, the RHS of (6.78) uniquely determines the finite-dimensional distributions of $\mathscr{X}_{t}^{(k)}$. Then, by the continuity of $\mathscr{X}_{t}^{(k)}$, we conclude the uniqueness of limiting point. We refer to [11] for more details on how to proceed for the case $k=2$.

\section{Appendix}

\subsection{Carré-du-champ}

Proposition 7.1. Consider an interacting particles system with generator

$$
L f(\eta)=\sum_{\eta^{\prime}} c\left(\eta, \eta^{\prime}\right)\left(f\left(\eta^{\prime}\right)-f(\eta)\right)
$$

the following is an alternative formulation for its carré-du-champ

$$
\Gamma(f)(\eta)=\sum_{\eta^{\prime}} c\left(\eta, \eta^{\prime}\right)\left(f\left(\eta^{\prime}\right)-f(\eta)\right)^{2} .
$$

Proof. By definition we have

$$
\begin{aligned}
\Gamma(f)(\eta) & =\sum_{\eta^{\prime}} c\left(\eta, \eta^{\prime}\right)\left(f\left(\eta^{\prime}\right)^{2}-f(\eta)^{2}\right) \\
& -2 f(\eta) \sum_{\eta^{\prime}} c\left(\eta, \eta^{\prime}\right)\left(f\left(\eta^{\prime}\right)-f(\eta)\right) \\
& =\sum_{\eta^{\prime}} c\left(\eta, \eta^{\prime}\right)\left(f\left(\eta^{\prime}\right)^{2}-f(\eta)^{2}\right) \\
& -\sum_{\eta^{\prime}} c\left(\eta, \eta^{\prime}\right)\left(2 f(\eta) f\left(\eta^{\prime}\right)-2 f(\eta)^{2}\right) \\
& =\sum_{\eta^{\prime}} c\left(\eta, \eta^{\prime}\right)\left(f\left(\eta^{\prime}\right)^{2}-f(\eta)^{2}-2 f(\eta) f\left(\eta^{\prime}\right)+2 f(\eta)^{2}\right) \\
& =\sum_{\eta^{\prime}} c\left(\eta, \eta^{\prime}\right)\left(f\left(\eta^{\prime}\right)^{2}-2 f(\eta) f\left(\eta^{\prime}\right)+f(\eta)^{2}\right) \\
& =\sum_{\eta^{\prime}} c\left(\eta, \eta^{\prime}\right)\left(f\left(\eta^{\prime}\right)-f(\eta)\right)^{2}
\end{aligned}
$$

that concludes the proof.

\subsection{Tightness criterium}

In this section we state a well known criterium for tightness extracted from [8]:

Theorem 7.2. Let $(\Omega, \mathscr{F})$ be a measurable space with right-continuous filtrations $\left\{\mathscr{F}_{t}^{n}\right\}_{t \geq 0}$ and probability measures $\mathbb{P}_{n}(\cdot), n \in \mathbb{N}$. Let $\left\{\mathscr{Y}_{t}^{n}\right\}_{t \geq 0}$ be an $\mathscr{F}_{t}^{n}$-adapted process with 
paths in $D\left([0, \infty), S^{\prime}\left(\mathbb{R}^{k}\right)\right)$ and let us also suppose that there exists, for each $\varphi \in S\left(\mathbb{R}^{k}\right)$, $\mathscr{F}_{t}^{n}$-predictable processes $\gamma_{1}^{n}(\cdot, \varphi), \gamma_{2}^{n}(\cdot, \varphi)$ such that:

$$
M_{t}^{n}(\varphi):=\mathscr{Y}_{t}^{n}(\varphi)-\int_{0}^{t} \gamma_{1}^{n}(s, \varphi) d s
$$

and

$$
M_{t}^{n}(\varphi)^{2}-\int_{0}^{t} \gamma_{2}^{n}(s, \varphi) d s
$$

are martingales. Assume further that it holds:

CI: for $t_{0} \geq 0$ and $\varphi \in S\left(\mathbb{R}^{k}\right)$ :

$$
\sup _{n \in \mathbb{N}} \sup _{0 \leq t \leq t_{0}} \mathbb{E}_{n}\left(\mathscr{Y}_{t}^{n}(\varphi)^{2}\right)<\infty
$$

and for $i \in\{1,2\}$ :

$$
\sup _{n \in \mathbb{N}} \sup _{0 \leq t \leq t_{0}} \mathbb{E}_{n}\left(\gamma_{i}^{n}(t, \varphi)^{2}\right)<\infty
$$

CII: for every $\varphi \in S\left(\mathbb{R}^{k}\right)$ there exists a sequence $\delta(t, \varphi, n)$ converging to zero as $n \rightarrow 0$ such that:

$$
\lim _{n \rightarrow \infty} \mathbb{P}_{n}\left(\sup _{0 \leq s \leq t}\left|\mathscr{Y}_{s}^{n}(\varphi)-\mathscr{Y}_{s-}^{n}(\varphi)\right| \geq \delta(t, \varphi, n)\right)=0
$$

then the family of laws $\left\{Q^{n}\right\}_{n \in \mathbb{N}}$, induced by $\left\{\mathscr{Y}_{t}^{n}\right\}_{t \geq 0}$ on $D\left([0, \infty), S^{\prime}\left(\mathbb{R}^{k}\right)\right)$ under $\mathbb{P}_{n}$, is a tight family and any weak limit point is supported by $C\left([0, \infty), S^{\prime}\left(\mathbb{R}^{k}\right)\right)$.

\section{References}

[1] Enrique Daniel Andjel, Invariant measures for the zero range processes, Ann. Probab. 10 (1982), no. 3, 525-547. MR-659526

[2] Sigurd Assing, A limit theorem for quadratic fluctuations in symmetric simple exclusion, Stochastic Process. Appl. 117 (2007), no. 6, 766-790. MR-2327838

[3] Mario Ayala, Gioia Carinci, and Frank Redig, Quantitative Boltzmann-Gibbs principles via orthogonal polynomial duality, J. Stat. Phys. 171 (2018), no. 6, 980-999. MR-3805584

[4] Gioia Carinci, Cristian Giardinà, and Frank Redig, Consistent particle systems and duality, 2019. arXiv:1907.10583

[5] Theodore S Chihara, An introduction to orthogonal polynomials, Courier Corporation, 2011.

[6] A. De Masi, N. Ianiro, A. Pellegrinotti, and E. Presutti, A survey of the hydrodynamical behavior of many-particle systems, Nonequilibrium phenomena, II, Stud. Statist. Mech., XI, North-Holland, Amsterdam, 1984, pp. 123-294. MR-757003

[7] Anna De Masi and Errico Presutti, Mathematical methods for hydrodynamic limits, Lecture Notes in Mathematics, vol. 1501, Springer-Verlag, Berlin, 1991. MR-1175626

[8] P. A. Ferrari, E. Presutti, and M. E. Vares, Nonequilibrium fluctuations for a zero range process, Ann. Inst. H. Poincaré Probab. Statist. 24 (1988), no. 2, 237-268. MR-953119

[9] Dmitri Finkelshtein, Yuri Kondratiev, Eugene Lytvynov, and Maria João Oliveira, An infinite dimensional umbral calculus, J. Funct. Anal. 276 (2019), no. 12, 3714-3766. MR-3957997

[10] Chiara Franceschini and Cristian Giardina, Stochastic duality and orthogonal polynomials, Sojourns in Probability Theory and Statistical Physics-III, Springer, 2019, pp. 187-214.

[11] Patrícia Gonçalves and Milton Jara, Quadratic fluctuations of the symmetric simple exclusion, ALEA Lat. Am. J. Probab. Math. Stat. 16 (2019), no. 1, 605-632. MR-3949272

[12] Claude Kipnis and Claudio Landim, Scaling limits of interacting particle systems, Grundlehren der Mathematischen Wissenschaften [Fundamental Principles of Mathematical Sciences], vol. 320, Springer-Verlag, Berlin, 1999. MR-1707314 
Higher order fluctuation fields

[13] Roelof Koekoek and Rene F Swarttouw, The askey-scheme of hypergeometric orthogonal polynomials and its q-analogue 1996, arXiv:math/9602214

[14] Frank Redig and Federico Sau, Factorized duality, stationary product measures and generating functions, J. Stat. Phys. 172 (2018), no. 4, 980-1008. MR-3830295

Acknowledgments. The authors would like to thank Federico Sau for helpful discussions; The authors also would like to thank valuable comments from anonymous reviewers. 


\section{Electronic Journal of Probability Electronic Communications in Probability}

\section{Advantages of publishing in EJP-ECP}

- Very high standards

- Free for authors, free for readers

- Quick publication (no backlog)

- Secure publication $\left(\mathrm{LOCKSS}^{1}\right)$

- Easy interface (EJMS²)

\section{Economical model of EJP-ECP}

- Non profit, sponsored by $\mathrm{IMS}^{3}, \mathrm{BS}^{4}$, ProjectEuclid ${ }^{5}$

- Purely electronic

\section{Help keep the journal free and vigorous}

- Donate to the IMS open access fund ${ }^{6}$ (click here to donate!)

- Submit your best articles to EJP-ECP

- Choose EJP-ECP over for-profit journals

\footnotetext{
${ }^{1}$ LOCKSS: Lots of Copies Keep Stuff Safe http://www. lockss.org/

${ }^{2}$ EJMS: Electronic Journal Management System http://www.vtex.lt/en/ejms.html

${ }^{3}$ IMS: Institute of Mathematical Statistics http://www.imstat.org/

${ }^{4}$ BS: Bernoulli Society http://www. bernoulli-society.org/

${ }^{5}$ Project Euclid: https://projecteuclid.org/

${ }^{6}$ IMS Open Access Fund: http://www.imstat.org/publications/open.htm
} 